\title{
Long non-coding RNA HOMER3-AS1 drives hepatocellular carcinoma progression via modulating the behaviors of both tumor cells and macrophages
}

\author{
Jian $\mathrm{Pu}^{1,4}$, Wenchuan $\mathrm{Li}^{1,4}$, Anmin Wang ${ }^{2}$, Ya Zhang ${ }^{2}$, Zebang $\mathrm{Qin}^{2}$, Zuoming $\mathrm{Xu}^{1}$, Jianchu Wang ${ }^{1}$, Yan Lu ${ }^{1}$, Qianli Tang (iD ${ }^{1 凶}$ and \\ Huamei Wei (iD) ${ }^{3 凶}$
}

(c) The Author(s) 2021

The crosstalk between cancer cells and tumor microenvironment plays critical roles in hepatocellular carcinoma (HCC). The identification of long non-coding RNAs (IncRNAs) mediating the crosstalk might promote the development of new therapeutic strategies against HCC. Here, we identified a IncRNA, HOMER3-AS1, which is over-expressed in HCC and correlated with poor survival of HCC patients. HOMER3-AS1 promoted HCC cellular proliferation, migration, and invasion, and reduced HCC cellular apoptosis. Furthermore, HOMER3-AS1 promoted macrophages recruitment and M2-like polarization. In vivo, HOMER3-AS1 significantly facilitated HCC progression. Mechanism investigations revealed that HOMER3-AS1 activated Wnt/ $\beta$-catenin signaling via upregulating HOMER3. Functional rescue experiments revealed that HOMER3/Wnt/ $\beta$-catenin axis mediated the roles of HOMER3-AS1 in promoting HCC cellular malignant phenotypes. Furthermore, colony stimulating factor-1 (CSF-1) was also identified as a critical downstream target of HOMER3-AS1. HOMER3-AS1 increased CSF-1 expression and secretion. Blocking CSF-1 reversed the roles of HOMER3-AS1 in inducing macrophages recruitment and M2 polarization. Furthermore, positive correlations between HOMER3-AS1 and HOMER3 expression, HOMER3-AS1 and CSF-1 expression, and HOMER3-AS1 expression and M2-like macrophages infiltration were found in human HCC tissues. In summary, our findings demonstrated that HOMER3-AS1 drives HCC progression via modulating the behaviors of both tumor cells and macrophages, which are dependent on the activation of HOMER3/Wnt/ $\beta$-catenin axis and CSF-1, respectively. HOMER3-AS1 might be a promising prognostic and therapeutic target for HCC.

Cell Death and Disease (2021)12:1103; https://doi.org/10.1038/s41419-021-04309-z

\section{INTRODUCTION}

Liver cancer is the sixth most commonly diagnosed malignancy and the third leading cause of malignancy-related death worldwide, with about 905,677 newly diagnosed cases and 830,180 liver cancer-caused deaths [1]. The main subtype of liver cancer is hepatocellular carcinoma (HCC), which accounts for $80 \%-90 \%$ of all liver cancer cases [2]. Although modern multidisciplinary therapies have been developed to fight against HCC, including surgical resection, liver transplantation, ablation, transcatheter arterial chemoembolization (TACE), molecular targeted therapies, and immunotherapy, only modest improvement in prognoses of HCC patients has been achieved [3]. Therefore, identifying molecular alterations in HCC that are responsible for HCC tumorigenesis and progression and developing novel more effective treatments are urgently needed [4-6].

The molecular alterations of HCC are very complex, with multiple genetic and epigenetic aberrations [7-9]. The ENCODE consortium shows that more than $80 \%$ of the human genome are transcribed into RNAs, whereas less than $2 \%$ of the human genome lastly encodes proteins [10]. Thus, the protein non-coding transcripts are significantly more diverse than protein-coding mRNAs [11-13]. Long non-coding RNAs (IncRNAs) are the most diverse class of non-coding transcripts, which also have diversely regulatory roles in various pathophysiological processes [14-19]. Several IncRNAs have been revealed to play critical roles in HCC $[20,21]$. IncRNA GPC3-AS1 was reported to enhance HCC cellular proliferation and migration [22]. MAGI2-AS3 was found to suppress HCC cellular malignant phenotype [23]. CASC9 depletion decreased HCC cellular viability [24]. IncRNA-ATB promoted HCC invasion-metastasis cascade [25].

The progression of HCC is not only regulated by malignant phenotypes of themselves, but also by tumor microenvironment (TME) [26-28]. Immune checkpoints inhibitors which enhance antitumor immune responses of $\mathrm{T}$ cells have been approved to treat HCC [29]. Apart from T cells, tumor-associated macrophages (TAMs) are another class of critical components of TME [8, 30]. Increasing evidences revealed that TAMs have M2-like phenotypes and contribute to tumor progression [27, 31]. Increased levels of M2-like TAMs in HCC were also revealed to be correlated with the poor prognosis of HCC patients [32]. Compared with the M2-like

\footnotetext{
${ }^{1}$ Department of Hepatobiliary Surgery, Affiliated Hospital of Youjiang Medical University for Nationalities, Baise, China. ${ }^{2}$ Graduate College of Youjiang Medical University for

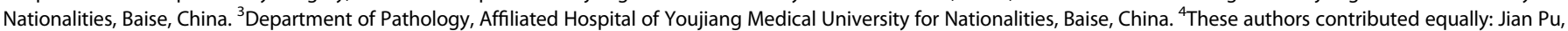
Wenchuan Li. ${ }^{凶}$ email: tang_qianli@yeah.net; weihuamei@yeah.net Edited by Professor Roberto Mantovani
}

Received: 1 July 2021 Revised: 7 October 2021 Accepted: 7 October 2021 Published online: 23 November 2021 
phenotype, macrophages can also be polarized to M1-like phenotypes, which frequently show antitumor activities [33, 34]. Although accumulating studies have identified several factors inducing $M 1$ or $M 2$ polarization of macrophages, such as the induction of M2 phenotype by IL-4, IL-10, TGF- $\beta$, and colonystimulating factor 1 (CSF-1), the contributions of IncRNAs to M2 polarization of TAMs are still largely unknown [35, 36].

In this study, we identified a poor prognosis-correlated IncRNA HOMER3-AS1 in HCC via analyzing the cancer genome atlas (TCGA) liver hepatocellular carcinoma (LIHC). The expression and clinical relevance of HOMER3-AS1 were further investigated in our HCC cohort. In vitro and in vivo functional experiments were undertaken to explore the biological roles of HOMER3-AS1 in HCC. The molecular mechanisms underlying the roles of HOMER3-AS1 in HCC were also investigated.

\section{MATERIALS AND METHODS \\ Bioinformatics analyses}

The correlation between gene expression and overall survival of HCC patients based on the RNA sequencing expression data of HCC tissues from the cancer genome atlas (TCGA) liver hepatocellular carcinoma (LIHC) project was analyzed by the online in silico tool GEPIA (http://gepia.cancerpku.cn/). Gene expression correlation in HCC tissues based on the TCGA LIHC dataset was calculated by GEPIA (http://gepia.cancer-pku.cn/). The genes which have similar expression patterns with HOMER3-AS1 in HCC based on the TCGA LIHC dataset were searched by GEPIA (http://gepia. cancer-pku.cn/).

\section{Clinical samples}

Sixty-eight pairs of HCC tissues and adjacent noncancerous liver tissues were acquired at the Affiliated Hospital of Youjiang Medical University for Nationalities from HCC patients who received surgical resection with written informed consents. All tissues were diagnosed by two experienced pathologists. The clinicopathological characteristics of these 68 cases were presented in Table 1. This study was performed following the Declaration of Helsinki and approved by the Ethics Committee of Affiliated Hospital of Youjiang Medical University for Nationalities.

\section{Cell cultures and reagents}

Human immortalized liver cell lines THLE-2 and THLE-3, and human HCC cell lines SK-HEP-1 and SNU-398 were acquired from American Type Culture Collection (ATCC). Human HCC cell line Huh7 and monocytic THP-1 cells were acquired from the National Collection of Authenticated Cell Cultures of the Chinese Academy of Sciences (Shanghai, China). THLE-2 and THLE-3 cells were cultured using BEGM Bullet Kit (Lonza, Walkersville, MD, USA) following the provided instructions. SK-HEP-1 cells were cultured in Eagle's Minimum Essential Medium (Invitrogen, Carlsbad, CA USA) added with 10\% fetal bovine serum (FBS, Invitrogen). SNU-398 and THP-1 cells were cultured in RPMI 1640 medium (Invitrogen) added with 10\% FBS. Huh7 cells were cultured in Dulbecco's modified Eagle's medium (Invitrogen) added with $10 \%$ FBS. All cells were grown at $37^{\circ} \mathrm{C}$ containing $5 \% \mathrm{CO}_{2}$. The cells were authenticated using STR profiles. All cells were routinely tested as mycoplasma-free. Where indicated, cells were treated with $10 \mu \mathrm{M}$ ICG-001 (Selleck, Houston, TX, USA), $0.2 \mu \mathrm{g} / \mathrm{mL}$ Anti-CSF-1 (AF216, R\&D Systems, Minneapolis, MN, USA), or $100 \mathrm{ng} / \mathrm{ml}$ phorbol-12-myristate-13-acetate (PMA, Sigma Aldrich, St. Louis, MO, USA).

\section{Quantitative real-time polymerase chain reaction (qRT-PCR)}

The total RNA from tissues and cells was extracted using TRlzol Reagent (Invitrogen). After quantification by UV-visible spectrophotometry, the RNA was reversely transcribed into complementary DNA (cDNA) using the M-MLV Reverse Transcriptase (Invitrogen) and random primers. qRT-PCR was undertaken using the TB Green Premix Ex Taq II (TaKaRa, Tokyo, Japan) on StepOnePlus Real-Time PCR System (Applied Biosystems, Foster City, CA, USA). The primer sequences were $5^{\prime}$-ACACCTCTTCTGCCTCCTC- ${ }^{\prime}$ (sense) and 5'-TGGCTTACATCTGTTATCCC-3' (anti-sense) for HOMER3-AS1, 5'-GCCGAGTITTCGCACTG-3' (sense) and 5'-GAATCTCCTGGTCCTTGGTTT-3' (anti-sense) for HOMER3, 5'-CTTCCCCTACCCTCTCAA-3' (sense) and 5'-CGATTTCTTCCTCATCTTCT-3' (anti-sense) for MYC, 5'-ACCGTGCTG TGTGCTGTG-3' (sense) and 5'-TCTCCTTGAGTTTGGCTTCT-3' (anti-sense) for
Table 1. Correlation between HOMER3-AS1 expression levels and clinicopathological characteristics in HCC.

\begin{tabular}{|c|c|c|c|c|c|}
\hline \multirow[t]{2}{*}{ Feature } & \multirow[t]{2}{*}{ Number } & \multicolumn{2}{|c|}{ HOMER3-AS1 } & \multirow[t]{2}{*}{$x^{2}$} & \multirow[t]{2}{*}{$p$-value } \\
\hline & & low & high & & \\
\hline Age & & & & 1.503 & 0.220 \\
\hline$>50$ & 39 & 22 & 17 & & \\
\hline$\leq 50$ & 29 & 12 & 17 & & \\
\hline Gender & & & & 0.637 & 0.425 \\
\hline Male & 61 & 29 & 32 & & \\
\hline Female & 7 & 5 & 2 & & \\
\hline HBs antigen & & & & 0.360 & 0.549 \\
\hline Positive & 54 & 26 & 28 & & \\
\hline Negative & 14 & 8 & 6 & & \\
\hline Liver cirrhosis & & & & 0.856 & 0.355 \\
\hline With & 13 & 8 & 5 & & \\
\hline Without & 55 & 26 & 29 & & \\
\hline BCLC stage & & & & 3.886 & 0.049 \\
\hline $0-A$ & 40 & 24 & 16 & & \\
\hline B-C & 28 & 10 & 18 & & \\
\hline AFP (ng/ml) & & & & 6.071 & 0.014 \\
\hline$>20$ & 40 & 15 & 25 & & \\
\hline$\leq 20$ & 28 & 19 & 9 & & \\
\hline Differentiation & & & & 0.183 & 0.669 \\
\hline I-II & 6 & 4 & 2 & & \\
\hline III-IV & 62 & 30 & 32 & & \\
\hline Encapsulation & & & & 4.798 & 0.091 \\
\hline Complete & 8 & 6 & 2 & & \\
\hline Not complete & 41 & 22 & 19 & & \\
\hline No & 19 & 6 & 13 & & \\
\hline Microvascular invasion & & & & 3.985 & 0.046 \\
\hline Absent & 42 & 25 & 17 & & \\
\hline Present & 26 & 9 & 17 & & \\
\hline
\end{tabular}

$p$-value was acquired by Pearson chi-square tests.

MMP7, 5'-CAGTGGCAATGAGGATGAC-3' (sense) and 5'-AGATGAAGGGAAA GAAGGTG-3' (anti-sense) for IL-1b, 5'-ATTGAGGTCATGGTGGAT-3' (sense) and 5'-GGAGAAGTAGGAATGTGGA-3' (anti-sense) for IL-12b, 5'-CTCAG CCTCTTCTCCTTCCT-3' (sense) and 5'-CTGGTTATCTCTCAGCTCCAC-3' (antisense) for TNF-a, $5^{\prime}$-GTGGAGCAGGTGAAGAATG-3' (sense) and 5'-GAAATG GGGGTTGAGGTATC-3' (anti-sense) for IL-10, 5'-AGCAGAGTTTGGTCAGGG-3' (sense) and 5'-GGCTTITTGTGGGGTTTTC-3' (anti-sense) for CD163, 5'-CGCC AAGTCCAGAACCAT-3' (sense) and 5'-TCTCAAGCAGACCAGCCT-3' (antisense) for ARG1, 5'-CCCTGCTGTTGTTGGTCTG-3' (sense) and 5'-GCATTGG GGGTGTTATCTCTG-3' (anti-sense) for CSF-1, 5'-GTCGGAGTCAACGGATTTG3' (sense) and 5'-TGGGTGGAATCATATTGGAA-3' (anti-sense) for GAPDH. GAPDH was employed as an endogenous control. Relative quantification was calculated by the comparative $\mathrm{Ct}$ method.

\section{Plasmids construction and transfection}

HOMER3-AS1 full-length sequences were PCR-amplified with the primers 5'-CCCAAGCTTGGAACCCAGGCCCTTCC-3' (sense) and 5'-CCGCTCGAGCA AGTTCTGAATTTAAGGCAGG-3' (anti-sense). The PCR products were further inserted into the Hind III and Xho I sites of $\operatorname{pCDNA}^{\mathrm{TM}_{3.1}(+)}$ Vector (Invitrogen) to yield HOMER3-AS1 overexpression plasmid. Two pairs of CDNA oligonucleotides repressing HOMER3-AS1 were synthesized and inserted into the shRNA lentivirus expressing vector $\mathrm{pLV3/H1/GFP \& Puro}$ (GenePharma, Shanghai, China). The constructed plasmid was cotransfected with pGag/Pol, pRev, and pVSV-G (GenePharma) into HEK293FT cells to generate shRNA lentivirus repressing HOMER3-AS1. 
Scrambled non-targeting shRNA lentivirus were used as negative control (NC). The shRNA oligonucleotide sequences were 5'-GATCCGGCCC ATGTTCCCTAGAGTITCAAGAGAAACTCTAGGGAAACATGGGCCTIIITTG-3' (sense) and 5'-AATTCAAAAAAGGCCCATGTTTCCCTAGAGTTTCTCTTGAA AACTCTAGGGAAACATGGGCCG-3' (anti-sense) for shRNA-HOMER3-AS1-1, 5'-GATCCGGAGTITGATGACTCAGTACTITCAAGAGAAGTACTGAGTCATCAAA CTCCTITITTG-3' (sense) and 5'-AATTCAAAAAAGGAGTTTGATGACTCAGTA CTTCTCTTGAAAGTACTGAGTCATCAAACTCCG-3' (anti-sense) for shRNAHOMER3-AS1-2, 5'-GATCCGTTCTCCGAACGTGTCACGTTTCAAGAGAACGTGA CACGTTCGGAGAACTITITTG-3' (sense) and 5'-AATTCAAAAAAGTTCTCCG AACGTGTCACGTTCTCTTGAAACGTGACACGTTCGGAGAACG-3' (anti-sense) for shRNA-NC. HOMER3 coding sequences (CDS) were PCR-amplified with the primers 5'-CCCAAGCTTCCCTAGAGCCTGCCCATC-3' (sense) and 5'-CC GCTCGAGGGAATCGTTCATAGAAAACC-3' (anti-sense). The PCR products were further inserted into the Hind III and Xho I sites of pCDNA ${ }^{\mathrm{TM}_{3}} 3.1(+)$ Vector to yield the HOMER3 overexpression plasmid. One pairs of CDNA oligonucleotides repressing HOMER3 were synthesized and inserted into pLV3/H1/GFP\&Puro (GenePharma). The constructed plasmid was cotransfected with pGag/Pol, pRev, and pVSV-G into HEK-293FT cells to generate shRNA lentivirus repressing HOMER3. The shRNA oligonucleotide sequences were 5'-GATCCCAAACCAAGGACCAGGAGATTITCAAGAGAAAT CTCCTGGTCCTTGGTITGTTITITG-3' (sense) and 5'-AATTCAAAAAACAAACC AAGGACCAGGAGATTTCTCTTGAAAATCTCCTGGTCCTTGGTTTGG-3' (anti-sense) for shRNA-HOMER3. Plasmids transfection and co-transfection were undertaken with Lipofectamine 3000 (Invitrogen).

\section{Stable cell lines construction}

To construct HOMER3-AS1 stably overexpressed and control cells, HOMER3-AS1 overexpression plasmid or empty plasmid pCDNA3.1 was transfected into THLE-2, SK-HEP-1, and SNU-398 cells. The transfected cells were treated with $800 \mu \mathrm{g} / \mathrm{ml}$ neomycin $48 \mathrm{~h}$ after transfection for 4 weeks to select HOMER3-AS1 overexpressed cells. To construct HOMER3-AS1 stably silenced and control cells, SK-HEP-1, and Huh7 cells were infected with shRNA lentivirus repressing HOMER3-AS1 or scrambled non-targeting shRNA lentivirus. The infected cells were treated with $2 \mu \mathrm{g} / \mathrm{ml}$ puromycin $96 \mathrm{~h}$ after infection for four weeks to select HOMER3-AS1 silenced cells. To construct HOMER3 stably overexpressed and control HCC cells, HOMER3 overexpression plasmid or empty plasmid pcDNA3.1 was transfected into SK-HEP-1 cells. The transfected cells were treated with $800 \mu \mathrm{g} / \mathrm{ml}$ neomycin $48 \mathrm{~h}$ after transfection for 4 weeks to select HOMER3 overexpressed HCC cells. To construct HOMER3-AS1 overexpressed and concurrently HOMER3 depleted HCC cells, HOMER3-AS1 overexpressed SK-HEP-1 cells were infected with shRNA lentivirus repressing HOMER3. The infected cells were treated with $2 \mu \mathrm{g} / \mathrm{ml}$ puromycin and $800 \mu \mathrm{g} / \mathrm{ml}$ neomycin $96 \mathrm{~h}$ after infection for four weeks to select HOMER3-AS1 overexpressed and concurrently HOMER3-AS1 silenced cells.

\section{Cell proliferation, apoptosis, migration, and invasion assays} Cell Counting Kit-8 (CCK-8) and 5-ethynyl-2'-deoxyuridine (EdU) incorporation experiments were undertaken to measure cell proliferation. For CCK-8 experiments, 2000 indicated HCC cells re-suspended in $100 \mu \mathrm{L}$ media were plated onto a 96-well plate. After culturing for $4 \mathrm{~d}, 10 \mu \mathrm{L}$ CCK-8 reagents (Dojindo, Kumamoto, Japan) were added to each well. After culture for another $2 \mathrm{~h}$, a microplate reader (BioTek, Winooski, VT, USA) was used to measure the absorbance at $450 \mathrm{~nm}$ to indicate the number of viable cells. For EdU incorporation experiments, indicated HCC cells were treated with $50 \mu \mathrm{M}$ EdU (RiboBio, Guangzhou, China) for $2 \mathrm{~h}$. After being fixed in $4 \%$ paraformaldehyde for $30 \mathrm{~min}$ and permeabilized using $0.5 \%$ TritonX-100 for $10 \mathrm{~min}$, the cells were stained with Apollo dye solution (RiboBio). The cell nucleus was further stained using DAPI. The number of EdU-positive and proliferative cells was detected using a fluorescence microscope (Carl Zeiss, Oberkochen, Germany). Cell apoptosis was detected using the Caspase-3 Activity Assay Kit (Cell Signaling Technology, Danvers, MA, USA) strictly following the provided protocol. Cell migration and invasion were evaluated by transwell migration and invasion assays as we previously described [23].

\section{Co-culture assay}

PMA-stimulated THP-1 cells were added into the upper chamber of $8 \mu \mathrm{m}$ pore transwell inserts (Corning, NY, USA). SK-HEP-1 cells with HOMER3-AS1 overexpression or silencing were plated into the lower chamber. After culture for $48 \mathrm{~h}$, THP-1 cells remaining in the upper chamber were removed. The migrated THP-1 cells were fixed, stained, and detected using a microscope. SK-HEP-1 cells with HOMER3-AS1 overexpression or silencing were plated into the upper chamber of $0.4 \mu \mathrm{m}$ pore transwell inserts (Corning). PMA-stimulated THP-1 cells were plated into the lower chamber. After co-culture for $96 \mathrm{~h}$, THP-1 cells were collected to extract RNA. Genes expression in THP-1 cells were detected by qRT-PCR. THP-1 cellular proliferation was detected by CCK- 8 experiments.

\section{Subcutaneous and orthotopic models}

Five-week-old male BALB/C athymic nude mice were purchased from Shanghai SLAC Laboratory Animal Co. and fed in Specific Pathogen Free (SPF) conditions. Luciferase-labelled SK-HEP-1 cells with HOMER3-AS1 overexpression or silencing were subcutaneously injected into nude mice. When the subcutaneous xenografts grew to about $0.7 \mathrm{~cm}$ in diameter, they were removed and cut into small pieces, which were then transplanted into the liver of nude mice. At the $14^{\text {th }}$ day after transplantation, the tumors were detected by bioluminescence imaging using IVIS ${ }^{@}$ Lumina II system (Caliper Life Sciences, Hopkinton, MA, USA). No statistical method was used to determine the sample size. The experiments were not randomized. The investigators performed the bioluminescence imaging were blinded to mouse allocation. The animal experiments were undertaken with the approval of the Animal Ethics Committee of Affiliated Hospital of Youjiang Medical University for Nationalities.

\section{Immunohistochemistry (IHC) and Immunofluorescence (IF)}

The liver orthotopic xenografts were used to perform IHC staining as previously described with primary antibodies against Ki67 (\#9027, 1:400, Cell Signaling Technology) or cleaved caspase-3 (\#9664, 1:1000, Cell Signaling Technology) [37]. Human HCC tissues were used to perform IHC staining with primary antibodies against CD163 (\#93498, 1:500, Cell Signaling Technology) or HOMER3 (16624-1-AP, 1:200, Proteintech, Chicago, IN, USA). The liver orthotopic xenografts were used to perform IF as previously described with primary antibodies against F4/80 (\#30325, 1:400, Cell Signaling Technology) [35]. SK-HEP-1 cells with HOMER3-AS1 overexpression or silencing were used to perform IF as previously described with primary antibodies against $\beta$-catenin (\#8480, 1:100, Cell Signaling Technology) [25].

\section{Western blot of total protein and nuclear protein}

Total protein was extracted from SK-HEP-1 cells with HOMER3-AS1 stable overexpression or silencing using the RIPA Lysis Buffer (Beyotime, Shanghai, China) added with proteinase inhibitor PMSF (Beyotime). Nuclear protein was extracted from SK-HEP-1 cells with HOMER3-AS1 stable overexpression or silencing using the Nuclear and Cytoplasmic Protein Extraction Kit (Beyotime, Shanghai, China). The extracted total protein and nuclear protein was separated by sodium dodecyl sulfatepolyacrylamide gel electrophoresis and then transferred onto polyvinylidene fluoride membrane. After being blocked with 5\% skimmed milk, the membranes were incubated with primary antibodies against HOMER3 (ab97438, 1:1000, Abcam, Cambridge, MA, USA), GAPDH (ab8245, 1:10000, Abcam), $\beta$-catenin (\#8480, 1:1000, Cell Signaling Technology), or histone H3 (ab1791, 1:1000). After three washes, the membranes were incubated with IRDye $800 \mathrm{CW}$ Goat anti-Rabbit IgG (LiCor, Lincoln, NE, USA) or IRDye 680RD Goat anti-Mouse IgG (Li-Cor), followed by being scanned on an Odyssey infrared scanner (Li-Cor). GAPDH and histone $\mathrm{H} 3$ were used as loading control for total protein and nuclear protein, respectively.

\section{RNA fluorescence in situ hybridization (FISH)}

For in situ detection of HOMER3-AS1 in HCC tissues, the HOMER3-AS1 probes were designed and synthesized by Advanced Cell Diagnostics (Hayward, CA, USA). RNAscope Fluorescent Multiplex Detection Kit (Advanced Cell Diagnostics) was used to perform the hybridization and fluorescence detection.

\section{Dual-luciferase reporter assays}

Wild-type Wnt/ $\beta$-catenin reporter TOPFlash (Addgene, Watertown, MA USA) and pRL-TK (Promega, Madison, WI, USA) were co-transfected into SKHEP-1 cells with HOMER3-AS1 stable overexpression or silencing. PRL-TK encodes Renilla luciferase and was used as endogenous control. After culture for another $48 \mathrm{~h}$, the Firefly luciferase and Renilla luciferase activities were measured using the Dual-Luciferase Reporter Assay System 
a
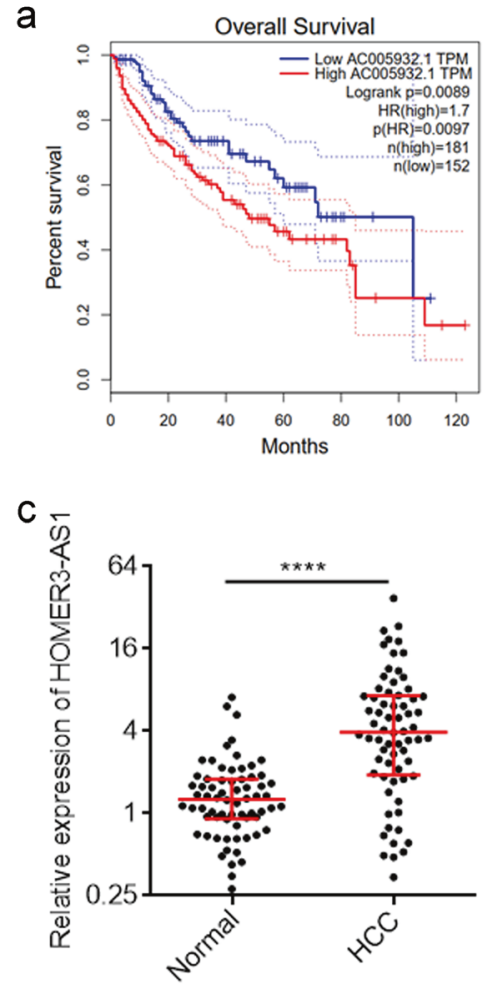

b
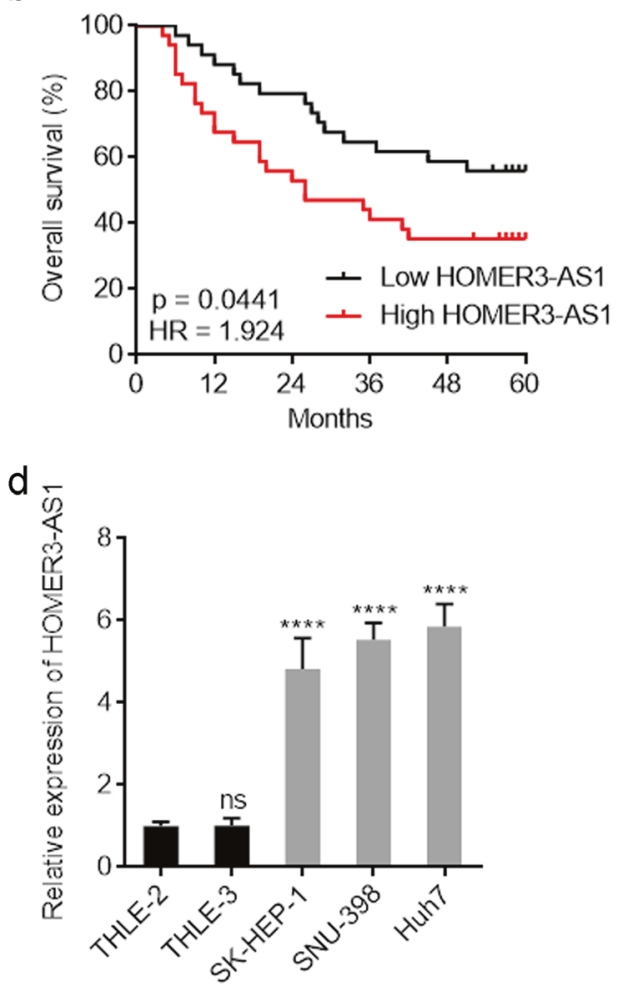

Fig. 1 The expression and clinical relevance of HOMER3-AS1 in HCC. a The correlation between HOMER3-AS1 (AC005932.1) expression and overall survival according to TCGA LIHC dataset, analyzed by GEPIA. b Kaplan-Meier survival analysis of the correlation between HOMER3-AS1 expression and overall survival in our HCC cohort. $n=68, p=0.0441, \mathrm{HR}=1.924$ by log-rank test. c HOMER3-AS1 expression in 68 pairs of HCC tissues and adjacent noncancerous liver tissues was measured by qRT-PCR. ${ }^{* * *} p<0.0001$ by Wilcoxon matched-pairs signed-rank test. d HOMER3-AS1 expression in immortalized human liver cell lines THLE-2 and THLE-3, and human HCC cell lines SK-HEP-1, SNU-398, and Huh7 were measured by qRT-PCR. Results are presented as mean \pm SD based on three independent experiments. ${ }^{* * *} p<0.0001$, ns, not significant, by one-way ANOVA followed by Dunnett's multiple comparisons test.

(Promega). The results were calculated as the ratio of Firefly luciferase activity to Renilla luciferase activity.

\section{Statistical analysis}

All statistical analyses were conducted using the GraphPad Prism 6.0 Software. The detailed statistical methods were shown in the figure and table legends. $p<0.05$ was considered statistically significant.

\section{RESULTS}

HOMER3-AS1 is correlated with advanced stage and poor prognosis in HCC

The correlation between HOMER3-AS1 expression levels and overall survival in HCC was analyzed by Gene Expression Profiling Interactive Analysis (GEPIA) (http://gepia.cancer-pku.cn/index. $\mathrm{html}$ ) based on the cancer genome atlas (TCGA) liver hepatocellular carcinoma (LIHC) dataset [38]. The results revealed that high HOMER3-AS1 (AC005932.1) expression was correlated with poor overall survival (Fig. 1a). To further test the correlation between HOMER3-AS1 expression levels and prognosis in $\mathrm{HCC}$, we collected 68 HCC tissues and measured HOMER3-AS1 expression by qRT-PCR. Consistently, high HOMER3-AS1 expression was correlated with poor overall survival in our HCC cohort (Fig. 1b). Analyses of the correlations between HOMER3-AS1 expression levels and clinicopathological characteristics revealed that high HOMER3-AS1 expression was correlated with advanced BCLC stage, high serum AFP concentration, and microvascular invasion (Table 1). In these 68 HCC cases, the expression of HOMER3-AS1 in paired adjacent noncancerous liver tissues was also measured. The results revealed that HOMER3-AS1 was significantly upregulated in HCC tissues compared with noncancerous liver tissues (Fig. 1c). Furthermore, the expression of HOMER3-AS1 in immortalized human liver cell lines THLE-2 and THLE-3, and human HCC cell lines SK-HEP-1, SNU-398, and Huh7 was measured. The result revealed that HOMER3-AS1 was also significantly upregulated in HCC cell lines compared with immortalized liver cell lines (Fig. 1d). The high expression and clinical relevance of HOMER3-AS1 in HCC implied that HOMER3-AS1 might be an oncogenic IncRNA in HCC.

HOMER3-AS1 promotes the malignant phenotype of HCC cells To explore the potential roles of HOMER3-AS1 in HCC, HOMER3AS1 was stably overexpressed in SK-HEP-1 and SNU-398 cells via transfection of HOMER3-AS1 overexpression plasmid (Fig. 2a). CCK-8 and EdU incorporation experiments revealed that overexpression of HOMER3-AS1 promoted HCC cellular proliferation (Fig. 2b, c). Caspase-3 activity assays revealed that overexpression of HOMER3-AS1 reduced HCC cellular apoptosis (Fig. 2d). Transwell migration and invasion experiments revealed that overexpression of HOMER3-AS1 promoted HCC cellular migration and invasion (Fig. 2e, f). Furthermore, HOMER3-AS1 was stably overexpressed in immortalized liver cell line THLE-2 (Supplementary Fig. 1a). CCK-8 and EdU incorporation experiments revealed that overexpression of HOMER3-AS1 also promoted THLE-2 cellular proliferation (Supplementary Fig. 1b, c). Caspase-3 activity assays revealed that overexpression of HOMER3-AS1 also reduced THLE-2 cellular apoptosis (Supplementary Fig. 1d). Transwell migration and invasion experiments revealed that overexpression of HOMER3-AS1 also promoted HCC cellular migration and 
a

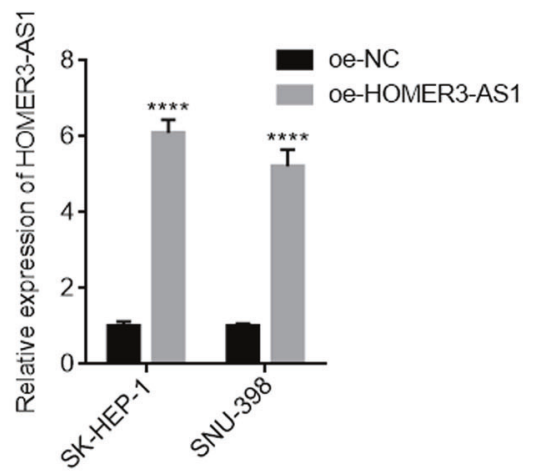

b

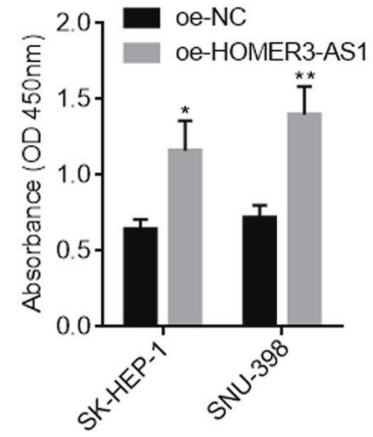

C

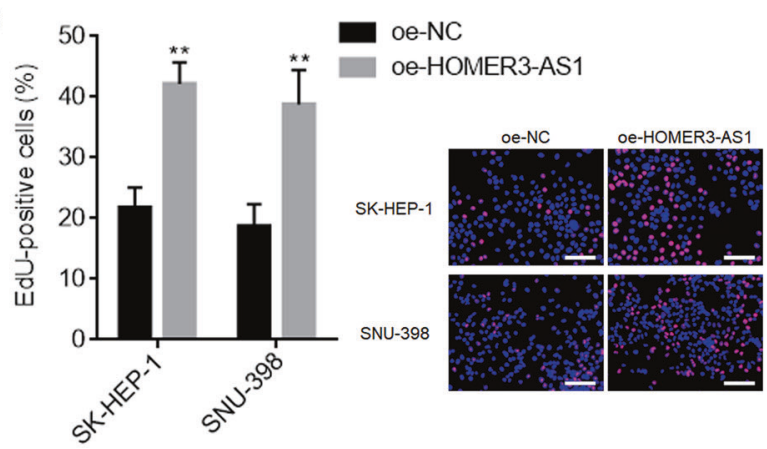

d

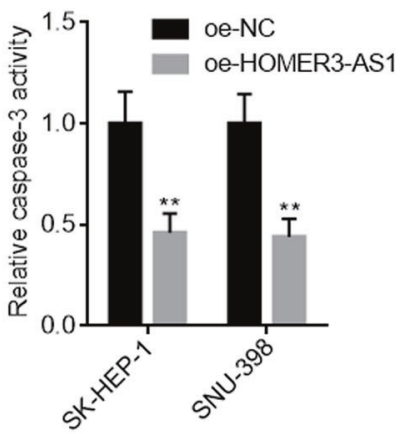

e

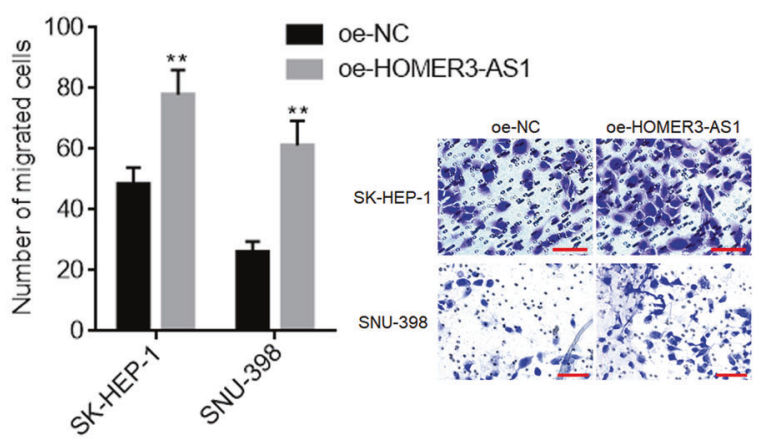

f

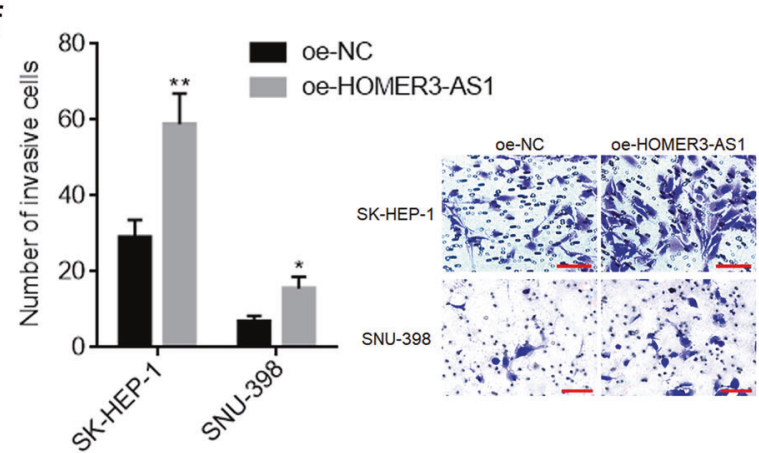

Fig. 2 Overexpression of HOMER3-AS1 promoted HCC cellular malignant phenotype. a HOMER3-AS1 expression in SK-HEP-1 and SNU-398 cells with HOMER3-AS1 stable overexpression was measured by qRT-PCR. b Cell proliferation of SK-HEP-1 and SNU-398 cells with HOMER3-AS1 stable overexpression was determined by CCK-8 experiments. c Cell proliferation of SK-HEP-1 and SNU-398 cells with HOMER3-AS1 stable overexpression was determined by EdU incorporation experiments. Scale bars, $100 \mu \mathrm{m}$. The red color indicates EdU-positive nucleuses and blue color indicates all cellular nucleuses. Results are shown as the ratio of EdU-positive nucleuses to all cellular nucleuses. $\mathbf{d}$ Cell apoptosis of SK-HEP-1 and SNU-398 cells with HOMER3-AS1 stable overexpression was determined by caspase-3 activity assays. e Cell migration of SK-HEP1 and SNU-398 cells with HOMER3-AS1 stable overexpression was determined by transwell migration assays. Scale bars, $100 \mu \mathrm{m} . \mathbf{f}$ Cell invasion of SK-HEP-1 and SNU-398 cells with HOMER3-AS1 stable overexpression was determined by transwell invasion assays. Scale bars, $100 \mu$ m. Results are presented as mean \pm SD based on three independent experiments. ${ }^{*} p<0.05,{ }^{* *} p<0.01,{ }^{* * *} p<0.0001$ by Student's $t$-test.

invasion (Supplementary Fig. 1e, f). These data suggested that overexpression of HOMER3-AS1 exerts oncogenic roles in HCC.

HOMER3-AS1 was stably silenced in SK-HEP-1 and Huh7 cells via infection of two independent HOMER3-AS1 specific shRNA lentiviruses (Supplementary Fig. 2a). CCK-8 and EdU incorporation experiments revealed that HOMER3-AS1 silencing restricted HCC cellular proliferation (Supplementary Fig. 2b, c). Caspase-3 activity assays revealed that HOMER3-AS1 silencing promoted HCC cellular apoptosis (Supplementary Fig. 2d). Transwell migration and invasion experiments revealed that HOMER3-AS1 silencing restricted HCC cellular migration and invasion (Supplementary Fig. $2 e, f)$. These data further support the oncogenic roles of HOMER3AS1 in HCC.

\section{HOMER3-AS1 promoted HCC tumor progression in vivo}

To further evaluate the roles of HOMER3-AS1 in HCC xenograft models, small pieces of subcutaneous tumors formed by luciferase labelled SK-HEP-1 cells with HOMER3-AS1 stable overexpression or silencing were orthotopically transplanted into the livers of nude mice. Bioluminescence imaging showed that the xenografts derived from HOMER3-AS1 overexpressed SK-HEP-1 cells were remarkably larger than those derived from control SK-HEP-1 cells (Fig. 3a). Conversely, the xenografts derived from HOMER3-AS1 silenced SK-HEP-1 cells were remarkably smaller than those derived from control SK-HEP-1 cells (Fig. 3b). Proliferation marker Ki67 IHC staining of orthotropic xenografts revealed that the xenografts derived from HOMER3-AS1 overexpressed SK-HEP-1 


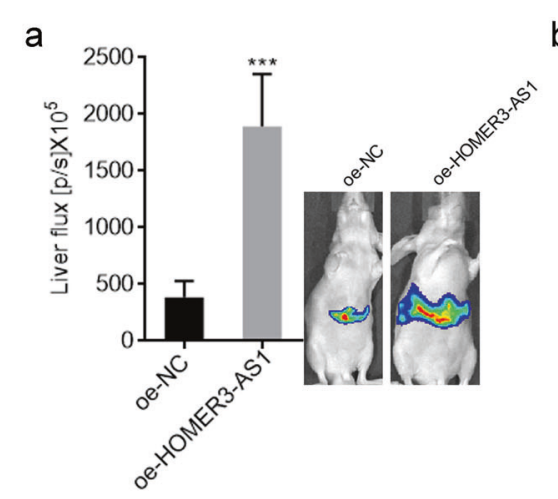

d

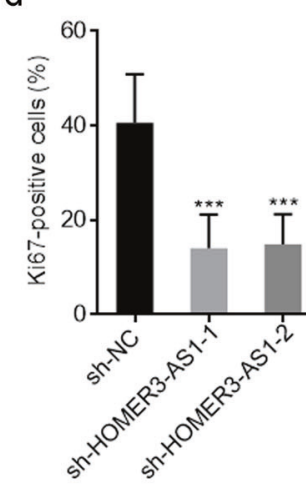

g

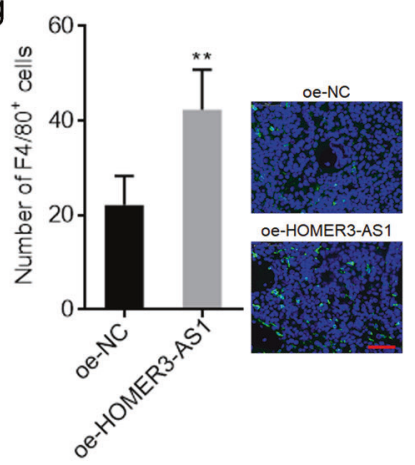

$\mathrm{h}$
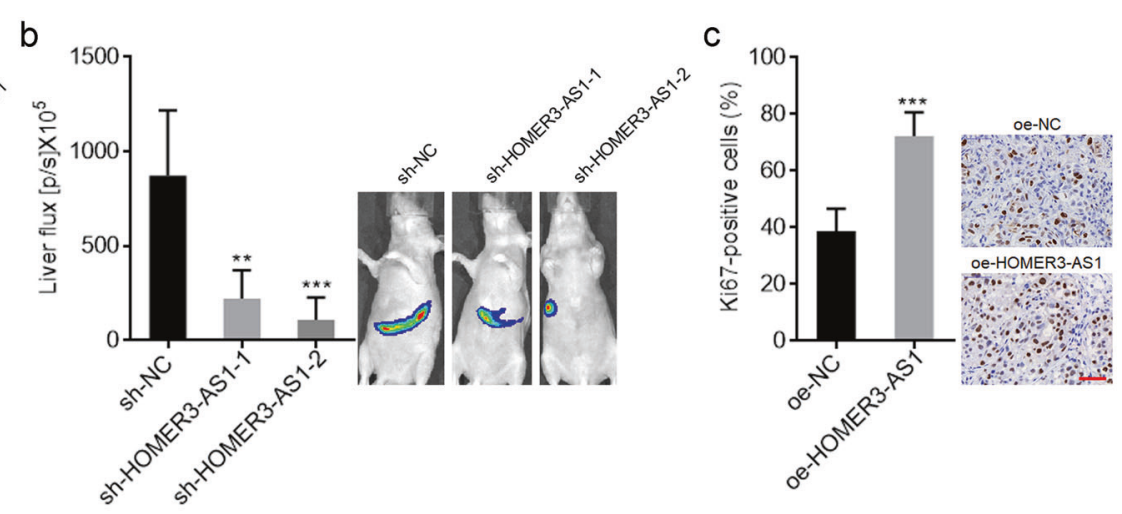

e $\%$

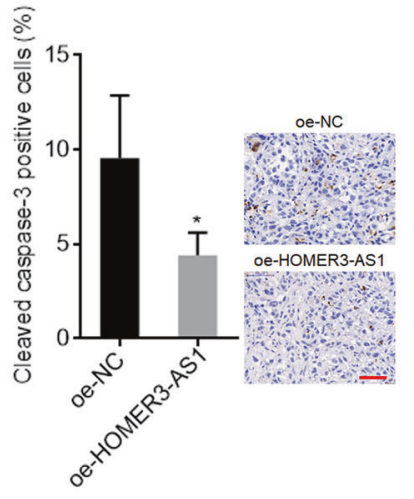

f
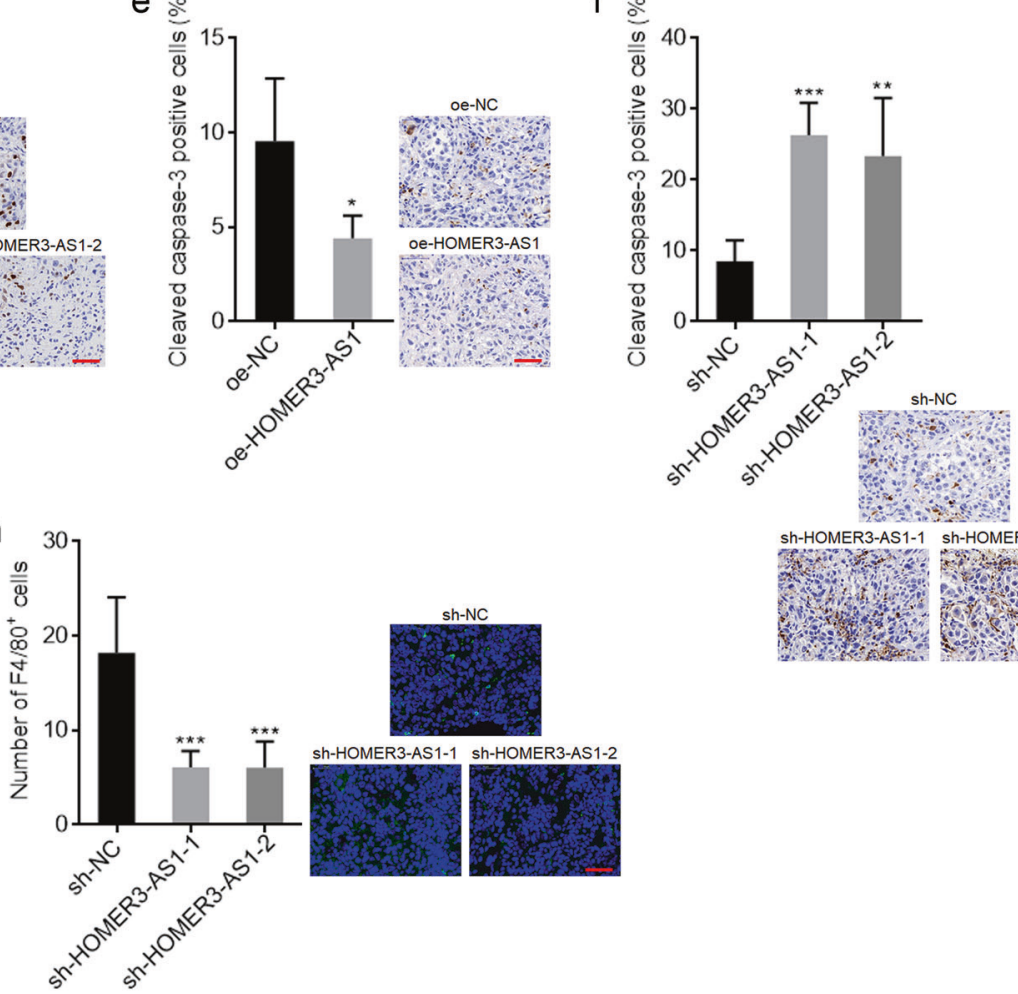

Fig. 3 HOMER3-AS1 promoted HCC tumor progression in vivo. a Bioluminescence imaging of liver tumors in mice at day 14 after inoculation with small pieces of subcutaneous tumors formed by luciferase labelled SK-HEP-1 cells with HOMER3-AS1 stable overexpression or control. b Bioluminescence imaging of liver tumors in mice at day 14 after inoculation with small pieces of subcutaneous tumors formed by luciferase labelled SK-HEP-1 cells with HOMER3-AS1 stable silencing or control. c Ki67 IHC staining of liver tumors formed by SK-HEP-1 cells with HOMER3-AS1 stable overexpression or control. Scale bars, $50 \mu \mathrm{m}$. d Ki67 IHC staining of liver tumors formed by SK-HEP-1 cells with HOMER3-AS1 stable silencing or control. Scale bars, $50 \mu \mathrm{m}$. e Cleaved caspase-3 IHC staining of liver tumors formed by SK-HEP-1 cells with HOMER3-AS1 stable overexpression or control. Scale bars, $50 \mu \mathrm{m}$. $\mathbf{f}$ Cleaved caspase-3 IHC staining of liver tumors formed by SK-HEP-1 cells with HOMER3-AS1 stable silencing or control. Scale bars, 50 mm. g F4/80 IF staining of liver tumors formed by SK-HEP-1 cells with HOMER3-AS1 stable overexpression or control. Scale bars, $50 \mu \mathrm{m}$. h F4/80 IF staining of liver tumors formed by SK-HEP-1 cells with HOMER3-AS1 stable silencing or control. Scale bars, $50 \mu \mathrm{m}$. Results are presented as mean \pm SD based on $n=5$ mice in each group. ${ }^{*} p<0.05$, ${ }^{* *} p<0.01$, ${ }^{* * *} p<$ 0.001 by Student's $t$-test $(\mathbf{a}, \mathbf{c}, \mathbf{e}, \mathbf{g})$ or one-way ANOVA followed by Dunnett's multiple comparisons test $(\mathbf{b}, \mathbf{d}, \mathbf{f}, \mathbf{h})$.

cells had more Ki67 positive cells than those derived from control SK-HEP-1 cells (Fig. 3c). Conversely, the xenografts derived from HOMER3-AS1 silenced SK-HEP-1 cells had less Ki67 positive cells than those derived from control SK-HEP-1 cells (Fig. 3d). Apoptosis marker cleaved caspase-3 IHC staining of orthotropic xenografts revealed that the xenografts derived from HOMER3-AS1 overexpressed SK-HEP-1 cells had less apoptotic cells than those derived from control SK-HEP-1 cells (Fig. 3e). Conversely, the xenografts derived from HOMER3-AS1 silenced SK-HEP-1 cells had more apoptotic cells than those derived from control SK-HEP-1 cells (Fig. 3f). Macrophage marker F4/80 IF staining of orthotropic xenografts revealed that the xenografts derived from HOMER3AS1 overexpressed SK-HEP-1 cells had more F4/80 ${ }^{+}$macrophages infiltration than those derived from control SK-HEP-1 cells (Fig. $3 \mathrm{~g}$ ). Conversely, the xenografts derived from HOMER3-AS1 silenced SKHEP- 1 cells had less $\mathrm{F} 4 / 80^{+}$macrophages infiltration than those derived from control SK-HEP-1 cells (Fig. 3h). Collectively, these data suggested that HOMER3-AS1 had oncogenic roles in vivo. HOMER3-AS1 not only promoted HCC cellular malignant phenotype, but also modulated tumor microenvironment. 


\section{HOMER3-AS1 upregulates HOMER3 and activates Wnt/ $\beta$-catenin signaling}

HOMER3-AS1 is transcribed in the anti-sense direction of HOMER3. Several antisense strand IncRNAs were revealed to regulate the expressions of their sense strand mRNAs $[22,37]$. Thus, we further investigated the potential effects of HOMER3-AS1 on HOMER3. qRT-PCR results revealed that HOMER3 mRNA levels were significantly upregulated after HOMER3-AS1 overexpression and significantly reduced after HOMER3-AS1 silencing (Fig. 4a, b). Western blot results revealed that HOMER3 protein levels were significantly upregulated after HOMER3-AS1 overexpression and significantly reduced after HOMER3-AS1 silencing (Fig. 4c, d). HOMER3 belongs to the HOMER family of density scaffolding proteins and was revealed to promote breast cancer progression via activating $W n t / \beta$-catenin signaling [39]. Thus, we further explored the potential effects of HOMER3-AS1 on Wnt/ $\beta$-catenin signaling in HCC. Subcellular fractionation and western blot experiments revealed that overexpression of HOMER3-AS1 increased nuclear $\beta$-catenin levels and while HOMER3-AS1 silencing reduced nuclear $\beta$-catenin levels (Fig. 4e, f). $\beta$-catenin IF staining also revealed that nuclear $\beta$-catenin increased after HOMER3-AS1 overexpression and reduced after HOMER3-AS1 silencing (Fig. 4g, h). Wnt/ $\beta$-catenin reporter TOPFlash luciferase activity assays revealed that overexpression of HOMER3-AS1 increased TOPFlash luciferase activity and while HOMER3-AS1 silencing reduced TOPFlash luciferase activity (Fig. $4 i, j$ ). Furthermore, Wnt/ $\beta$-catenin downstream targets MYC and MMP7 were upregulated after HOMER3-AS1 overexpression and downregulated after HOMER3-AS1 silencing (Fig. 4k, I). Collectively, these data suggested that HOMER3-AS1 upregulates HOMER3 and activates Wnt/ $\beta$-catenin signaling in HCC.

\section{HOMER3 expression is positively correlated with HOMER3-AS1} expression and poor prognosis in HCC

HOMER3 expression in the same 68 pairs of HCC tissues and adjacent noncancerous liver tissues were measured. Similar with HOMER3-AS1, HOMER3 was significantly upregulated in HCC tissues compared with noncancerous liver tissues (Supplementary Fig. 3a). The expression of HOMER3 was significantly positively correlated with that of HOMER3-AS1 in these 68 HCC tissues (Supplementary Fig. 3b). Similar with HOMER3-AS1, high HOMER3 expression was correlated with poor overall survival in this HCC cohort (Supplementary Fig. 3c). The remarkably positive correlation between HOMER3 and HOMER3-AS1 expression in HCC tissues was also confirmed in the TCGA LIHC dataset, analyzed by GEPIA (Supplementary Fig. 3d). Analyses of the TCGA LIHC dataset by GEPIA also revealed that high HOMER3 expression was correlated with poor overall survival in HCC (Supplementary Fig. 3e). Furthermore, the expressions of HOMER3-AS1 and HOMER3 in HCC tissues were detected by RNA FISH and IHC respectively. The results revealed that both HOMER3-AS1 and HOMER3 were mainly expressed in HCC cells, but not in stromal cells (Supplementary Fig. 3f), supporting clinical relevancy of HOMER3-AS1 and HOMER3 in HCC.

\section{HOMER3-AS1 promotes HCC cellular malignant phenotype via regulating HOMER3/Wnt/ $\beta$-catenin}

To explore whether HOMER3/Wnt/ $\beta$-catenin mediates the oncogenic roles of HOMER3-AS1 in HCC, we first detected the potential roles of HOMER3 in HCC. HOMER3 was stably overexpressed in SK-HEP-1 cells via transfection of the HOMER3 overexpression plasmid (Supplementary Fig. 4a). CCK-8 and EdU incorporation experiments revealed that overexpression of HOMER3 promoted HCC cellular proliferation (Supplementary Fig. 4b, c). Caspase- 3 activity assays revealed that overexpression of HOMER3 reduced HCC cellular apoptosis (Supplementary Fig. 4d). Transwell migration and invasion experiments revealed that overexpression of HOMER3 promoted HCC cellular migration and invasion (Supplementary Fig. 4e, f). HOMER3 was stably silenced in HOMER3-AS1 overexpressed SK-HEP-1 cells via infection of HOMER3 specific shRNA lentiviruses (Fig. 5a). CCK-8 and EdU incorporation experiments revealed that depletion of HOMER3 abolished the HOMER3-AS1-induced promotion of proliferation (Fig. 5b, c). Caspase-3 activity assays revealed that depletion of HOMER3 abolished the HOMER3-AS1-induced inhibition of apoptosis (Fig. 5d). Transwell migration and invasion experiments revealed that depletion of HOMER3 abolished the HOMER3-AS1-induced promotion of migration and invasion (Fig. 5e, f). Furthermore, HOMER3-AS1 overexpressed and control SK-HEP-1 cells were treated with $\mathrm{Wnt} / \beta$-catenin inhibitor ICG001 . The blocking of $W n t / \beta$-catenin signaling abolished the HOMER3-AS1-induced promotion of proliferation, migration, and invasion, and inhibition of apoptosis (Fig. $5 \mathrm{~g}-\mathrm{k}$ ). Collectively, these data suggested that HOMER3-AS1 promotes HCC cellular malignant phenotype via regulating HOMER3/Wnt/ $\beta$-catenin axis.

\section{HOMER3-AS1 induces infiltration and M2-like polarization of macrophages}

Given that the xenografts formed by HOMER3-AS1 overexpressed HCC cells had more macrophages infiltration, we further explored the potential influences of HOMER3-AS1 on macrophages using in vitro co-culture system by culturing HOMER3-AS1 overexpressed or silenced SK-HEP-1 cells with PMA-treated THP-1 cells (Fig. 6a). HOMER3-AS1 overexpressed SK-HEP-1 cells promoted the migration of macrophages compared with control SK-HEP-1 cells (Fig. 6a). Conversely, HOMER3-AS1 silenced SK-HEP-1 cells inhibited the migration of macrophages compared with control SK-HEP-1 cells (Fig. 6b). At the condition of co-culture, HOMER3-AS1 overexpressed SK-HEP-1 cells enhanced the proliferation of macrophages compared with control SK-HEP-1 cells (Fig. 6c). Conversely, HOMER3-AS1 silenced SK-HEP-1 cells restricted the proliferation of macrophages compared with control SK-HEP-1 cells (Fig. 6d). HOMER3-AS1 overexpressed SK-HEP-1 cells-stimulated macrophages exhibited reduced expression of M1 markers, IL-1b, IL$12 \mathrm{~b}$, and TNF-a, with increased expression of M2 markers, IL-10, CD163, and ARG1 (Fig. 6e). Conversely, HOMER3-AS1 silenced SKHEP-1 cells-stimulated macrophages exhibited increased expression of $\mathrm{M} 1$ markers, IL-1b, IL-12b, and TNF- $\mathrm{a}$, with reduced expression of M2 markers, IL-10, CD163, and ARG1 (Fig. 6f). Collectively, these data suggested that HOMER3-AS1 induces macrophages infiltration and M2-like polarization.

\section{CSF-1 upregulation is responsible for the recruitment and M2- like polarization of macrophages by HOMER3-AS1}

To elucidate the mechanisms mediating the roles of HOMER3AS1 in macrophages recruitment and polarization, we searched the genes with similar expression pattern with HOMER3-AS1 in HCC based on the TCGA LIHC dataset analyzed by GEPIA. CSF-1 ranked the first with a Pearson correlation coefficient of 0.72 (Supplementary Fig. 5a). Similar with HOMER3-AS1, high CSF-1 expression was correlated with poor overall survival in HCC based on the TCGA LIHC dataset analyzed by GEPIA (Supplementary Fig. 5b). CSF-1 expression in the same $68 \mathrm{HCC}$ tissues of our cohort were measured. The expression of CSF-1 was significantly positively correlated with that of HOMER3-AS1 in our HCC cohort (Supplementary Fig. 5c). Similar with HOMER3AS1, high CSF-1 expression was correlated with poor overall survival in this HCC cohort (Supplementary Fig. $5 d$ ). CSF-1 is frequently reported to be a critical regulator of macrophages generation, differentiation, and function [36, 40]. Thus, we further detected the correlation between HOMER3-AS1, CSF-1, and macrophages recruitment in HCC tissues. CD163 IHC staining was performed in these $68 \mathrm{HCC}$ tissues to indicate 


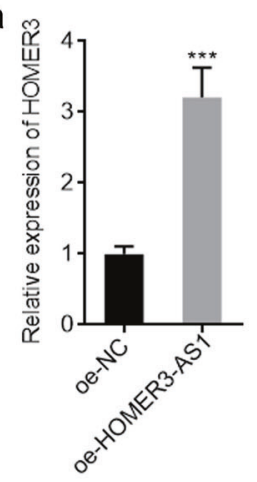

e

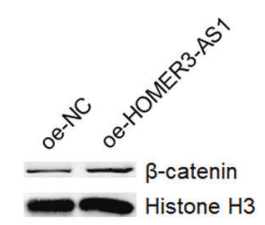

b

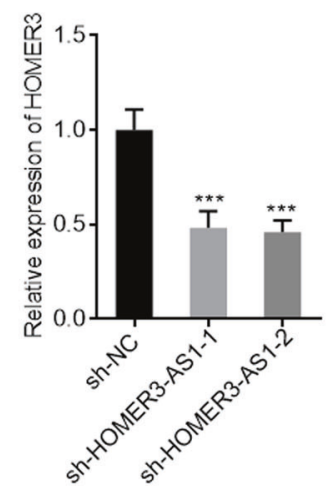

f

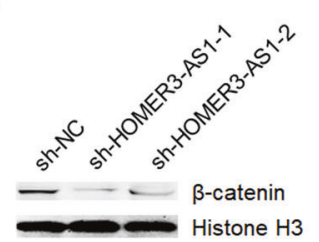

C

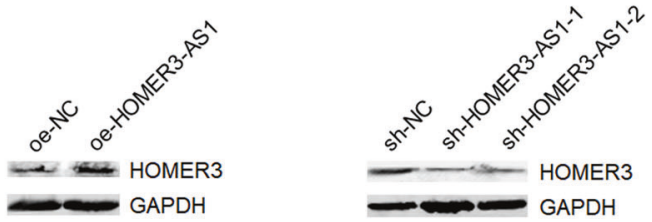

g

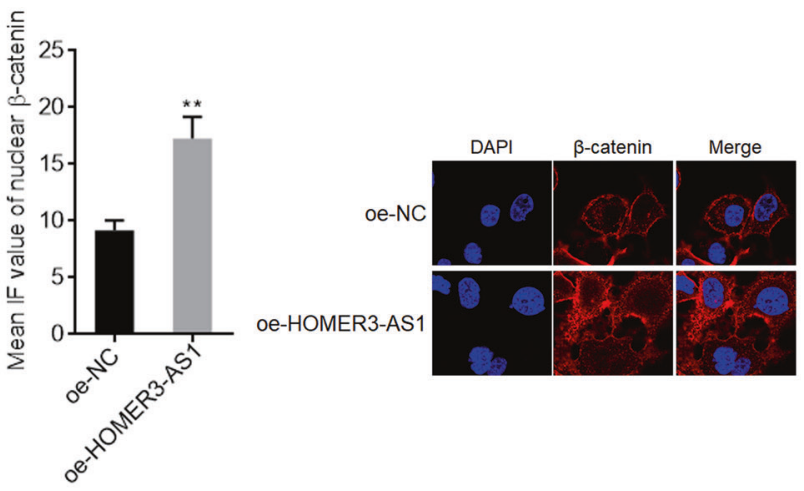

h

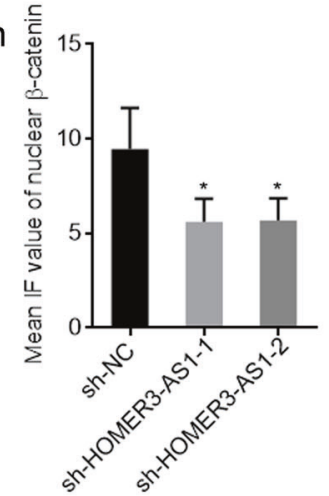

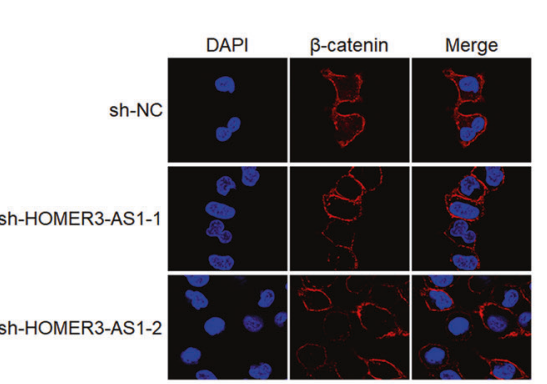

i

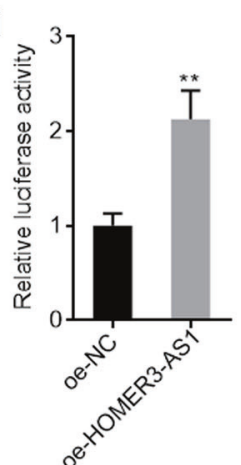

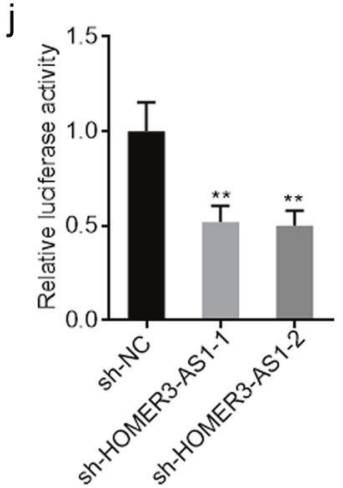

$\mathrm{k}$

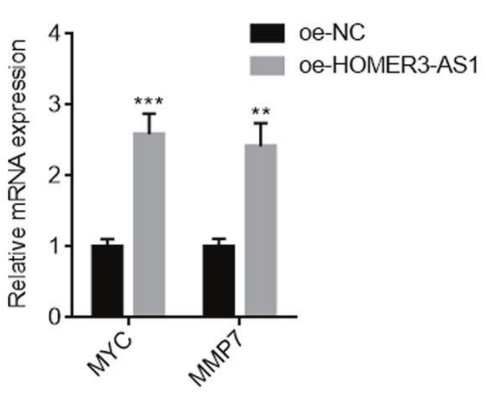

I

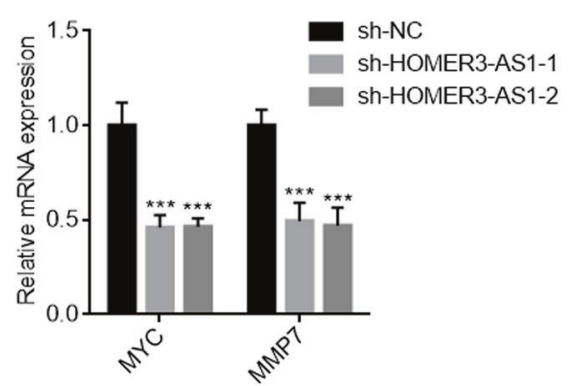

Fig. 4 HOMER3-AS1 increased HOMER3 expression and activated Wnt/ $\boldsymbol{\beta}$-catenin signaling. a HOMER3 mRNA levels in SK-HEP-1 cells with HOMER3-AS1 stable overexpression or control was measured by qRT-PCR. b HOMER3 mRNA levels in SK-HEP-1 cells with HOMER3-AS1 stable silencing or control were measured by qRT-PCR. c HOMER3 protein levels in SK-HEP-1 cells with HOMER3-AS1 stable overexpression or control was measured by western blot. $\mathbf{d}$ HOMER3 protein levels in SK-HEP-1 cells with HOMER3-AS1 stable silencing or control was measured by qRTPCR. e Nuclear $\beta$-catenin protein levels in SK-HEP-1 cells with HOMER3-AS1 stable overexpression or control was measured by western blot. f Nuclear $\beta$-catenin protein levels in SK-HEP-1 cells with HOMER3-AS1 stable silencing or control was measured by western blot. $\mathbf{g} \beta$-catenin expression in the nucleus of SK-HEP-1 cells with HOMER3-AS1 stable overexpression or control was detected by IF. $\mathbf{h} \beta$-catenin expression in the nucleus of SK-HEP-1 cells with HOMER3-AS1 stable silencing or control was detected by IF. $\mathbf{i} \beta$-catenin reporter TOPFlash and pRL-TK which encodes Renilla luciferase were transfected into SK-HEP-1 cells with HOMER3-AS1 stable overexpression or control. Dual-luciferase reporter assays were undertaken to determine $\beta$-catenin transcriptional activity. $\mathbf{j} \beta$-catenin reporter TOPFlash and pRL-TK were transfected into SKHEP-1 cells with HOMER3-AS1 stable silencing or control. Dual-luciferase reporter assays were undertaken to determine $\beta$-catenin transcriptional activity. k Wnt/ $\beta$-catenin downstream targets MYC and MMP7 expressions in SK-HEP-1 cells with HOMER3-AS1 stable overexpression or control. I Wnt/ $\beta$-catenin downstream targets MYC and MMP7 expressions in SK-HEP-1 cells with HOMER3-AS1 stable silencing or control. Results are presented as mean \pm SD based on three independent experiments. ${ }^{*} p<0.05,{ }^{* *} p<0.01,{ }^{* *} p<0.001$ by Student's $t$-test $(\mathbf{a}, \mathbf{g}, \mathbf{i}, \mathbf{k})$ or one-way ANOVA followed by Dunnett's multiple comparisons test $(\mathbf{b}, \mathbf{h}, \mathbf{j}, \mathbf{I})$. 
a

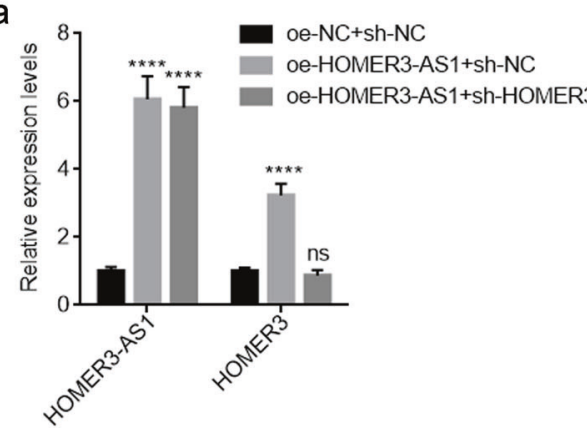

b

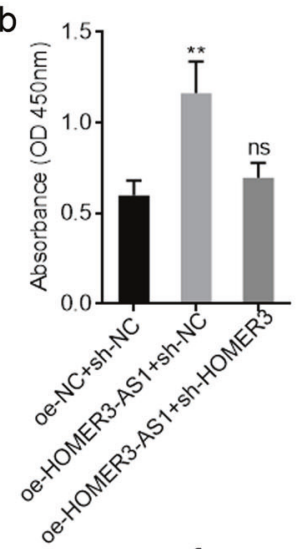

C

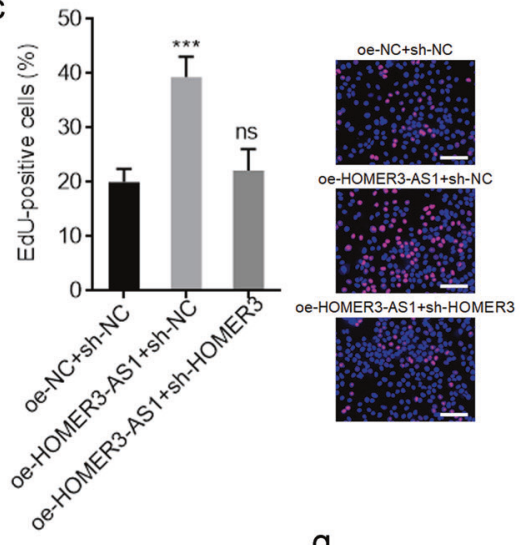

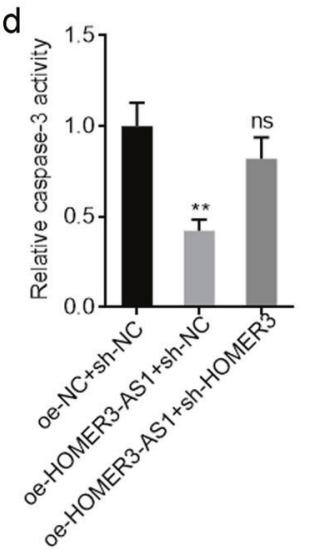

$\mathrm{h}$

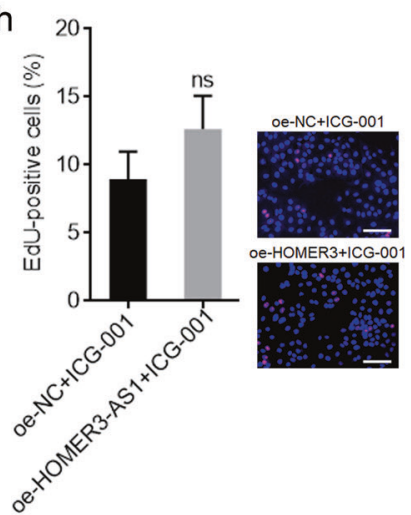

e
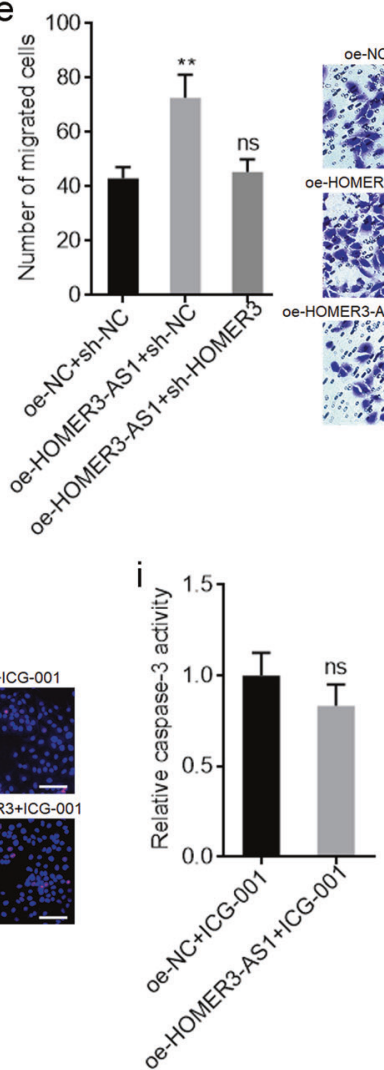

f

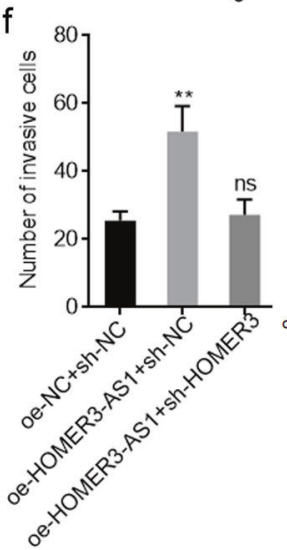

g
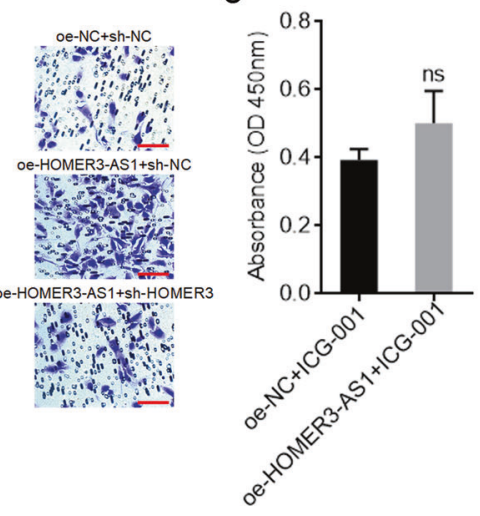
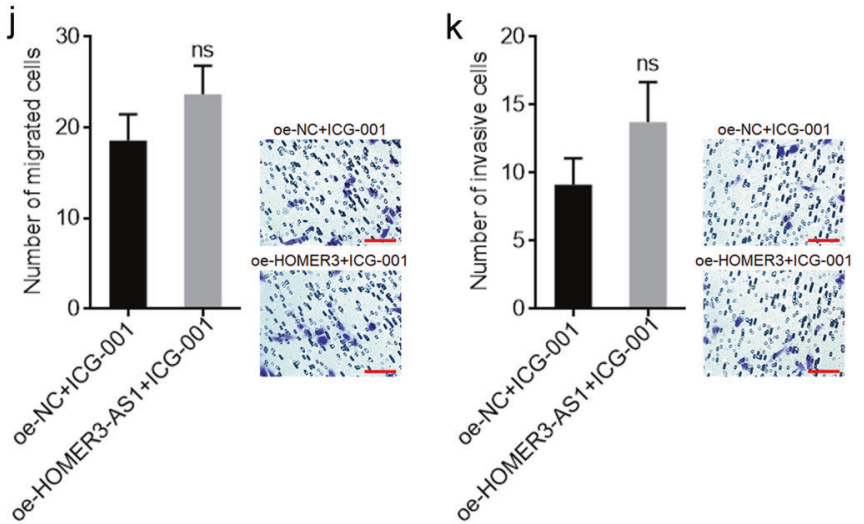

Fig. 5 Inhibition of HOMER3/Wnt/ $\beta$-catenin signaling abolished the oncogenic roles of HOMER3-AS1 in HCC. a HOMER3-AS1 and HOMER3 expression in SK-HEP-1 cells with HOMER3-AS1 overexpression and concurrent HOMER3 depletion. b Cell proliferation of SK-HEP-1 cells with HOMER3-AS1 overexpression and HOMER3 depletion was determined by CCK-8 experiments. c Cell proliferation of SK-HEP-1 cells with HOMER3-AS1 overexpression and HOMER3 depletion was determined by EdU incorporation experiments. Scale bars, $100 \mu \mathrm{m}$. $\mathbf{d}$ Cell apoptosis of SK-HEP-1 cells with HOMER3-AS1 overexpression and HOMER3 depletion was determined by caspase-3 activity assays. e Cell migration of SK-HEP-1 cells with HOMER3-AS1 overexpression and HOMER3 depletion was determined by transwell migration assays. Scale bars, 100 mm. f Cell invasion of SK-HEP-1 cells with HOMER3-AS1 overexpression and HOMER3 depletion was determined by transwell invasion assays. Scale bars, $100 \mu \mathrm{m}$. g Cell proliferation of HOMER3-AS1 overexpressed and control SK-HEP-1 cells treated with $10 \mu \mathrm{M}$ ICG-001 was determined by CCK-8 experiments. $\mathbf{h}$ Cell proliferation of HOMER3-AS1 overexpressed and control SK-HEP-1 cells treated with $10 \mu \mathrm{M}$ ICG-001 was determined by EdU incorporation experiments. Scale bars, $100 \mu \mathrm{m}$. i Cell apoptosis of HOMER3-AS1 overexpressed and control SK-HEP-1 cells treated with $10 \mu \mathrm{M}$ ICG-001 was determined by caspase-3 activity assays. $\mathbf{j}$ Cell migration of HOMER3-AS1 overexpressed and control SK-HEP-1 cells treated with $10 \mu \mathrm{M}$ ICG-001 was determined by transwell migration assays. Scale bars, $100 \mu \mathrm{m}$. k Cell invasion of HOMER3-AS1 overexpressed and control SK-HEP-1 cells treated with $10 \mu \mathrm{M}$ ICG-001 was determined by transwell invasion assays. Scale bars, $100 \mu \mathrm{m}$. Results are presented as mean \pm SD based on three independent experiments. ${ }^{* *} p<0.01,{ }^{* *} p<0.001,{ }^{* * * *} p<0.0001$, ns, not significant, by one-way ANOVA followed by Dunnett's multiple comparisons test (a-f) or Student's $t$-test $(\mathbf{g}-\mathbf{k})$.

macrophages infiltration and polarization. The results revealed that the HCC tissues with high levels of $\mathrm{CD} 163^{+}$macrophages also had higher HOMER3-AS1 and CSF-1 expression levels than those HCC tissues with low levels of $\mathrm{CD}_{163}{ }^{+}$macrophages (Supplementary Fig. 5e).
CSF-1 was significantly upregulated in SK-HEP-1 and SNU-398 cells with HOMER3-AS1 overexpression compared with control SKHEP-1 and SNU-398 cells (Fig. 7a). Conversely, the CSF-1 expression level was significantly reduced after HOMER3-AS1 silencing (Fig. 7b). Furthermore, ELISA results revealed that HCC 
a

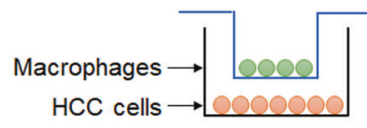

C

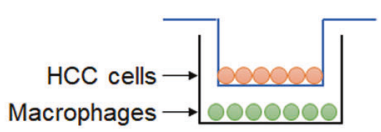

Macrophages $\rightarrow 000000$

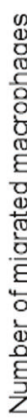

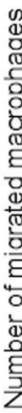

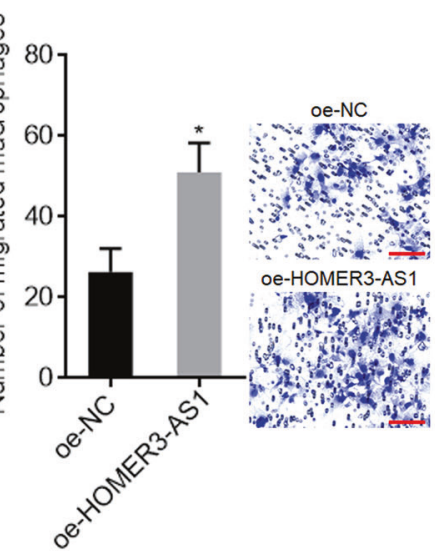

b

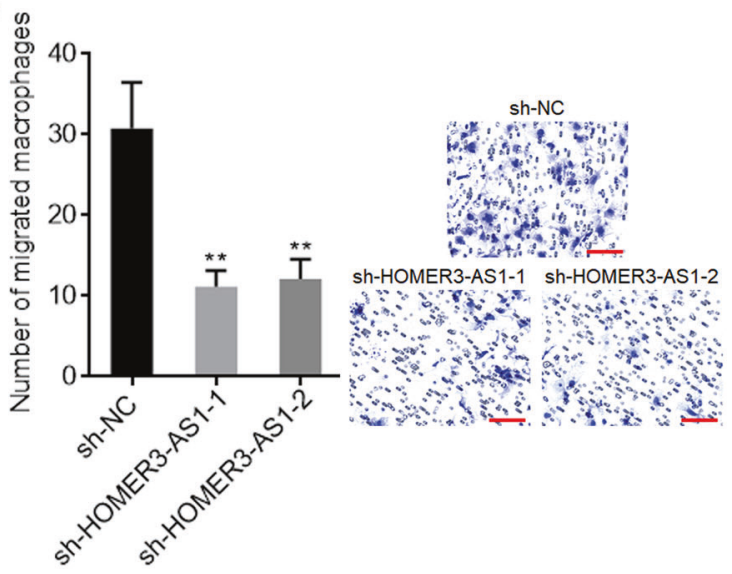

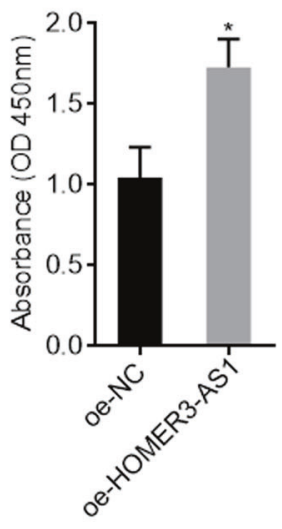

d

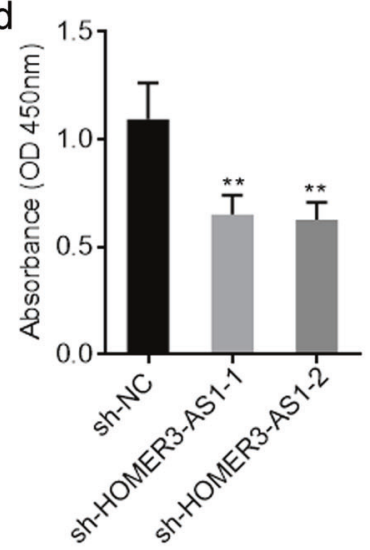

e

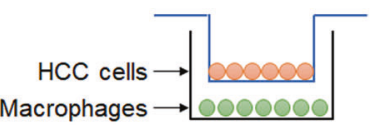

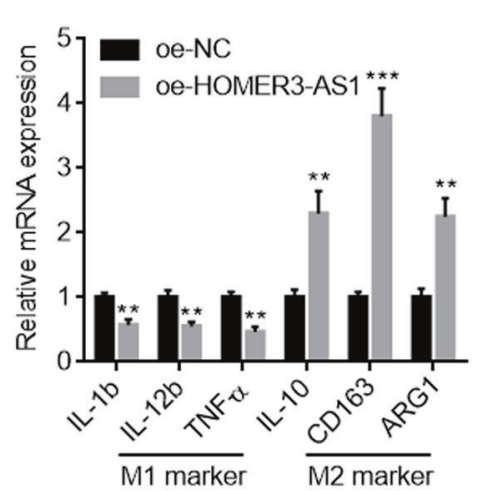

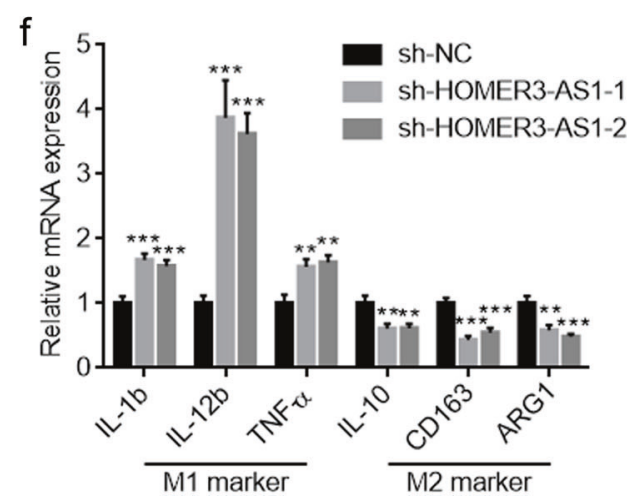

Fig. 6 HOMER3-AS1 promoted macrophages infiltration and M2-like polarization. a PMA-stimulated THP-1 cells were subjected to migration assays towards SK-HEP-1 cells with HOMER3-AS1 overexpression or control. b PMA-stimulated THP-1 cells were subjected to migration assays towards SK-HEP-1 cells with HOMER3-AS1 silencing or control. c Cell proliferation of PMA-stimulated THP-1 cells co-cultured with HOMER3-AS1 overexpressed or control SK-HEP-1 cells was determined by CCK-8 experiments. d Cell proliferation of PMA-stimulated THP1 cells co-cultured with HOMER3-AS1 silenced or control SK-HEP-1 cells was determined by CCK-8 experiments. e Expression of markers associated with M1 and M2 polarization in PMA-stimulated THP-1 cells co-cultured with HOMER3-AS1 overexpressed or control SK-HEP-1 cells was detected by qRT-PCR. f Expression of markers associated with M1 and M2 polarization in PMA-stimulated THP-1 cells co-cultured with HOMER3-AS1 silenced or control SK-HEP-1 cells was detected by qRT-PCR. Results are presented as mean \pm SD based on three independent experiments. ${ }^{*} p<0.05,{ }^{* *} p<0.01,{ }^{* *} p<0.001$ by Student's $t$-test $(\mathbf{a}, \mathbf{c}, \mathbf{e})$ or one-way ANOVA followed by Dunnett's multiple comparisons test $(b, d, f)$.

cells with HOMER3-AS1 overexpression had increased CSF-1 secretion compared with control cells (Fig. 7c). Conversely, HOMER3-AS1 silencing reduced CSF-1 secretion (Fig. 7d). To elucidate whether the induced secretion of CSF-1 by HOMER3-AS1 mediates the roles of HOMER3-AS1 in inducing macrophages infiltration and polarization, conditioned medium (CM) from SKHEP-1 cells with HOMER3-AS1 overexpression or control was used to stimulate PMA-treated THP-1 cells. Similar with co-culture, CM from HOMER3-AS1 overexpressed SK-HEP-1 cells also promoted macrophages migration, proliferation, and M2 polarization
(Fig. 7e-g). Anti-CSF-1 treatment reversed HOMER3-AS1-induced macrophages migration, proliferation, and M2 polarization (Fig. 7e-g). Collectively, these data suggested that HOMER3-AS1 upregulated CSF-1 expression and secretion, which further induced macrophages recruitment and M2-like polarization.

\section{DISCUSSION}

Increasing understandings of the crosstalk between tumor cells and tumor microenvironment (TME) have driven advances in 


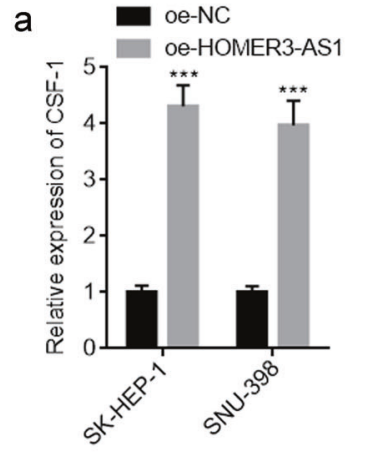

d
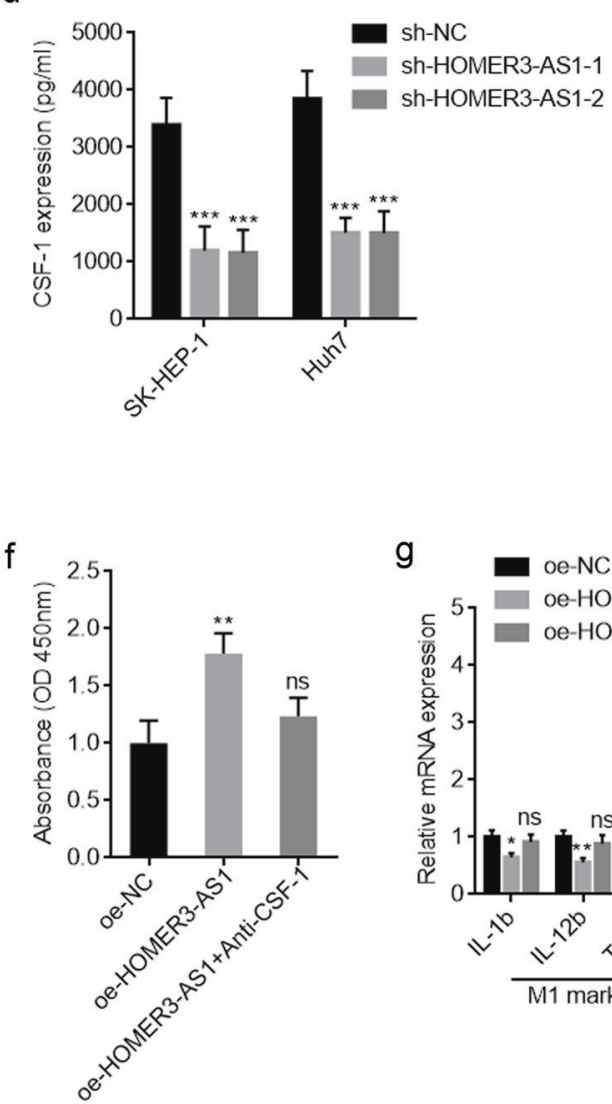

g
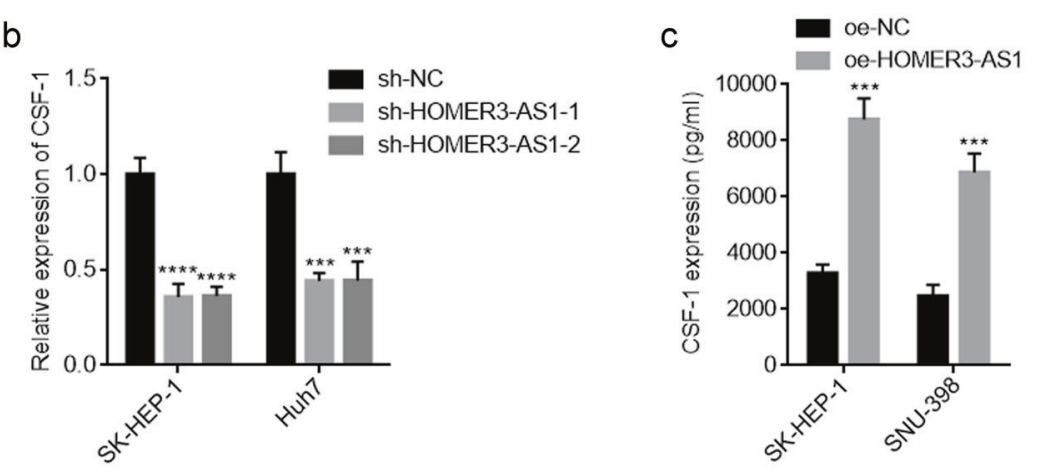

e
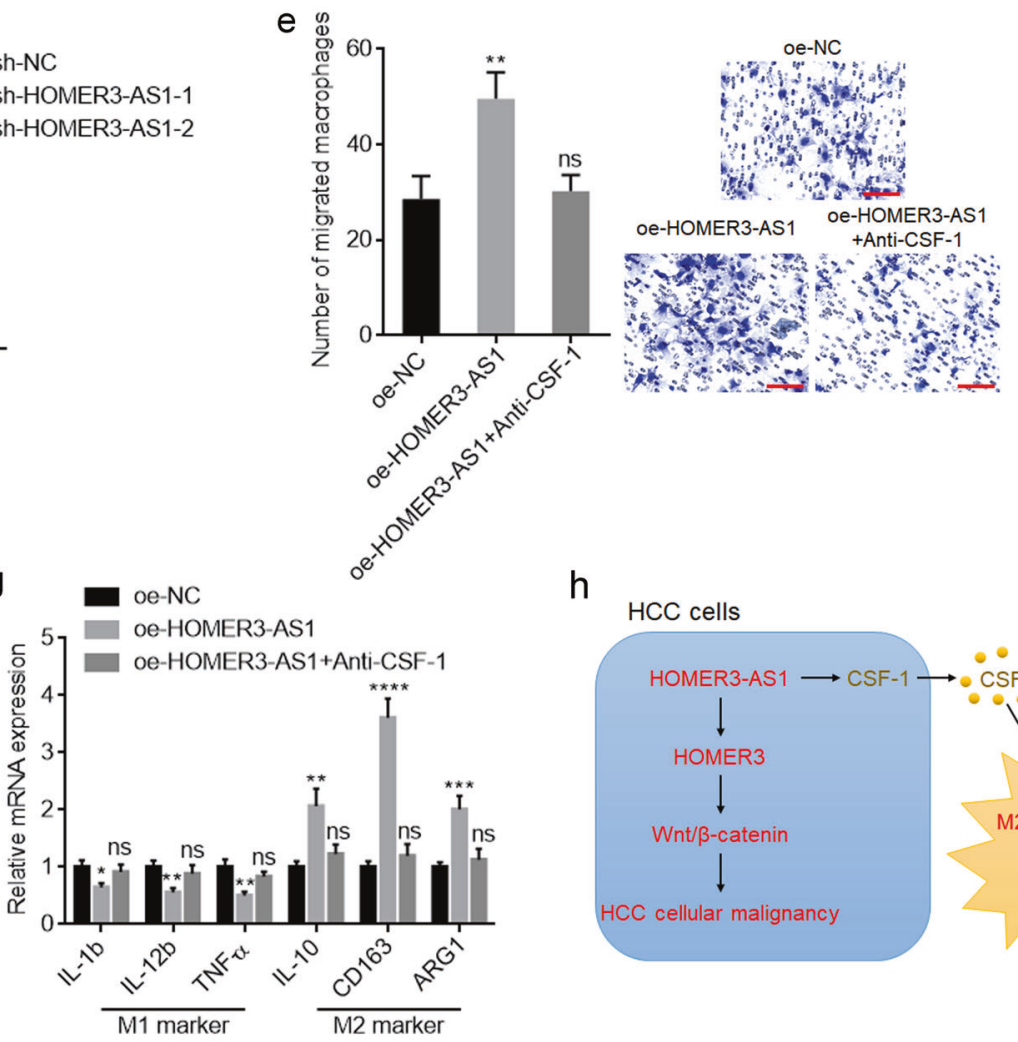

h

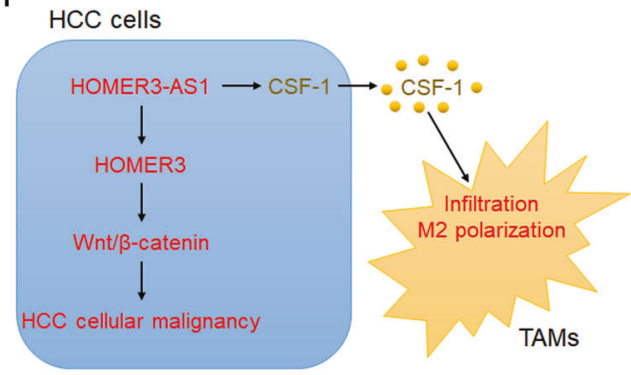

Fig. 7 HOMER3-AS1 promoted macrophages infiltration and M2-like polarization via upregulating CSF-1. a CSF-1 expression in SK-HEP-1 and SNU-398 cells with HOMER3-AS1 stable overexpression was measured by qRT-PCR. b CSF-1 expression in SK-HEP-1 and Huh7 cells with HOMER3-AS1 stable silencing was measured by qRT-PCR. c CSF-1 secretion from SK-HEP-1 and SNU-398 cells with HOMER3-AS1 stable overexpression was measured by ELISA. d CSF-1 secretion from SK-HEP-1 and Huh7 cells with HOMER3-AS1 stable silencing was measured by ELISA. e PMA-stimulated THP-1 cells were subjected to migration assays towards CM from HOMER3-AS1 overexpressed SK-HEP-1 cells treated with or without anti-CSF-1. f Cell proliferation of PMA-stimulated THP-1 cells after culture with CM from HOMER3-AS1 overexpressed SK-HEP-1 cells treated with or without anti-CSF-1. g Expression of markers associated with M1 and M2 polarization in PMA-stimulated THP-1 cells after culture with CM from HOMER3-AS1 overexpressed SK-HEP-1 cells treated with or without anti-CSF-1. $\mathbf{h}$ Schematic model of the roles of HOMER3-AS1 in regulating HCC cellular malignancy and TAMs infiltration and polarization. Results are presented as mean \pm SD based on three independent experiments. ${ }^{*} p<0.05,{ }^{* *} p<0.01,{ }^{* * *} p<0.001,{ }^{* * * *} p<0.0001$, ns, not significant, by Student's $t$-test (a, c) or one-way ANOVA followed by Dunnett's multiple comparisons test (b, $\mathbf{d}-\mathbf{g})$.

immunotherapy [41-44]. Immune checkpoints inhibitors, such as anti-PD-1 antibody nivolumab and pembrolizumab, have been approved for HCC treatment $[45,46]$. However, only about $14 \%$ of HCC patients are sensitive to anti-PD-1 antibody. Furthermore, frequent drug resistances to anti-PD-1 antibody were also found in clinical therapy [47]. Thus, further revealing the critical molecules driving HCC progression via mediating the crosstalk between HCC cells and TME would provide novel avenues for HCC therapy.
In this study, we identified a novel HCC-related IncRNA HOMER3-AS1. HOMER3-AS1 is located in chromosome 19p13.11. HOMER3-AS1 is transcribed in the anti-sense direction of HOMER3 and has 1041 nucleotides in length. Both RNA-sequencing data of the TCGA LIHC project and QRT-PCR detection of our HCC cohort revealed that high expression of HOMER3-AS1 is correlated with poor overall survival of HCC patients. Furthermore, our data also revealed that HOMER3-AS1 is upregulated in HCC tissues and correlated with advanced BCLC stage, high serum AFP 
concentration, and microvascular invasion. Therefore, these findings suggested HOMER3-AS1 as a potential prognostic biomarker for HCC. The positive correlation between HOMER3AS1 and AFP suggested that HOMER3-AS1 may be a diagnostic biomarker for HCC, which needs further investigation.

Gain- and loss-of functions experiments revealed that HOMER3AS1 has oncogenic roles in HCC. HOMER3-AS1 drives HCC progression in vitro and in vivo. HOMER3-AS1 enhances HCC cellular malignant phenotypes, including the promotion of HCC cellular proliferation, migration, and invasion, and the inhibition of HCC cellular apoptosis. Moreover, HOMER3-AS1 was also found to facilitate macrophages infiltration and M2-like polarization. Therefore, HOMER3-AS1 drives HCC progression not only by regulating the behaviors of HCC cells, but also by educating macrophages. Our findings also showed that depletion of HOMER3-AS1 significantly represses $\mathrm{HCC}$ progression in vitro and in vivo through reducing HCC cellular malignant phenotypes and macrophages recruitment and M2 polarization. HOMER3-AS1 represents a potential therapeutic target for HCC.

As a class of regulatory non-coding RNAs, IncRNAs exert their oncogenic or tumor-suppressive roles mainly through modulating critical oncogenic or tumor suppressive proteins and signaling pathways [24, 48, 49]. IncRNA PHAROH promotes HCC cellular proliferation and migration via upregulating MYC protein expression [48]. LncRNA MRCCAT1 promotes clear cell renal cell carcinoma metastasis through activating MAPK signaling [49]. IncRNA CASC9 enhances HCC cellular viability via activating AKT signaling [24]. Apart from MAPK and AKT signaling, other signaling pathways were also revealed to be involved in HCC, such as Wnt/ $\beta$-catenin, TGF- $\beta, N F-k B$, and Hedgehog signaling pathways [50-53]. Here, we found that HOMER3-AS1 activated Wnt/ $\beta$-catenin signaling via upregulating HOMER3. Wnt/ $\beta$-catenin signaling is frequently reported to be involved in stemness and malignant progression of HCC [54]. In this study, we also found that blocking of Wnt/ $\beta$-catenin signaling abolished the oncogenic roles of HOMER3-AS1 in HCC cellular malignant phenotypes. Anti-sense IncRNAs could modulate the expression of their sense strand genes at transcriptional, posttranscriptional, or translational levels [22, 37]. GPC3-AS1 epigenetically activates GPC3 transcription [22]. PXN-AS1-L enhances PXN mRNA stability, and while PXN-AS1-S represses PXN mRNA translation [37]. Here, we found that HOMER3-AS1 upregulates HOMER3 mRNA and protein levels. Thus, HOMER3-AS1 may transcriptionally activate HOMER3 expression or increase HOMER3 mRNA stability, which need further investigation.

Except for the roles of HOMER3-AS1 in enhancing HCC cellular malignant phenotypes, HOMER3-AS1 was also found to induce macrophages recruitment and M2-like polarization. Several molecules were reported to mediate the crosstalk between cancer cells and macrophages $[35,36,55]$. Tumor cells secrete sonic hedgehog to drive TAMs M2 polarization [55]. OPN derived from HCC cells facilitate macrophages migration and $\mathrm{M} 2$ polarization through activating the CSF-1/CSF1R pathway [35]. CircASAP1 promotes macrophage infiltration via modulating the miR-326/miR-532-5pCSF-1 pathway [36]. Combining bioinformatics analyses and experimental verification, we identified CSF-1 as the critical downstream target of HOMER3-AS1 and the mediator of the roles of HOMER3-AS1 in inducing macrophages recruitment and $\mathrm{M} 2$ polarization. The expression of CSF-1 is significantly positively correlated with that of HOMER3-AS1 in HCC tissues, verified in the TCGA LIHC dataset and our HCC cohort. The expression levels of CSF-1 and HOMER3-AS1 are both positively correlated with the number of CD163 positive macrophages. These clinical data supported the HOMER3-AS1/CSF-1/ macrophages regulatory axis. The detailed molecular mechanisms of how HOMER3-AS1 regulates CSF-1 need further investigation.

In summary, we identified IncRNA HOMER3-AS1 as a novel HCCrelated IncRNA, which is highly expressed in HCC tissues and correlated with poor survival of HCC patients. HOMER3-AS1 enhances HCC cellular malignant phenotypes via upregulating
HOMER3 and activating Wnt/ $\beta$-catenin signaling. HOMER3-AS1 facilitates macrophages infiltration and $\mathrm{M} 2$-like polarization via upregulating CSF-1 and inducing CSF-1 secretion (Fig. 7h). These findings suggested HOMER3-AS1 as a potential prognostic biomarker and therapeutic target for HCC.

\section{DATA AVAILABILITY}

The datasets generated and/or analyzed during the current study are available from the corresponding author on reasonable request.

\section{REFERENCES}

1. Sung, H, Ferlay, J, Siegel, RL, Laversanne, $M$, Soerjomataram, I, Jemal, $A$ et al. Global cancer statistics 2020: GLOBOCAN estimates of incidence and mortality worldwide for 36 cancers in 185 countries. CA Cancer J Clin. (2021);71:209-249.

2. Asrani SK, Devarbhavi H, Eaton J, Kamath PS. Burden of liver diseases in the world. J Hepatol. 2019;70:151-71.

3. Abou-Alfa GK, Meyer T, Cheng AL, El-Khoueiry AB, Rimassa L, Ryoo BY, et al. Cabozantinib in patients with advanced and progressing hepatocellular carcinoma. N. Engl J Med. 2018;379:54-63.

4. Mendez-Blanco C, Fondevila F, Garcia-Palomo A, Gonzalez-Gallego J, Mauriz JL. Sorafenib resistance in hepatocarcinoma: role of hypoxia-inducible factors. Exp Mol Med. 2018;50:1-9.

5. Lee G, Jeong YS, Kim DW, Kwak MJ, Koh J, Joo EW, et al. Clinical significance of APOB inactivation in hepatocellular carcinoma. Exp Mol Med. 2018;50:1-12.

6. Kim JH, Choi YK, Byun JK, Kim MK, Kang YN, Kim SH, et al. Estrogen-related receptor gamma is upregulated in liver cancer and its inhibition suppresses liver cancer cell proliferation via induction of p21 and p27. Exp Mol Med. 2016;48:e213.

7. Kudo Y, Sugimoto M, Arias E, Kasashima H, Cordes T, Linares JF, et al. PKClambda/ iota loss induces autophagy, oxidative phosphorylation, and NRF2 to promote liver cancer progression. Cancer Cell. 2020;38:247-62. e211

8. Zhao J, Li H, Zhao S, Wang E, Zhu J, Feng D, et al. Epigenetic silencing of miR-144/ 451a cluster contributes to HCC progression via paracrine HGF/MIF-mediated TAM remodeling. Mol Cancer. 2021;20:46.

9. Liu XN, Yuan JH, Wang TT, Pan W, Sun SH. An alternative POLDIP3 transcript promotes hepatocellular carcinoma progression. Biomed Pharmacother. 2017;89:276-83.

10. Djebali S, Davis CA, Merkel A, Dobin A, Lassmann T, Mortazavi A, et al. Landscape of transcription in human cells. Nature. 2012;489:101-8.

11. Hanniford D, Ulloa-Morales A, Karz A, Berzoti-Coelho MG, Moubarak RS, SanchezSendra $B$, et al. Epigenetic silencing of CDR1as drives IGF2BP3-mediated melanoma invasion and metastasis. Cancer Cell. 2020;37:55-70. e15

12. Esposito R, Bosch N, Lanzos A, Polidori T, Pulido-Quetglas C, Johnson R. Hacking the cancer genome: profiling therapeutically actionable long non-coding RNAs using CRISPR-Cas9 screening. Cancer Cell. 2019;35:545-57.

13. Song BW, Lee CY, Kim R, Kim WJ, Lee HW, Lee MY, et al. Multiplexed targeting of miRNA-210 in stem cell-derived extracellular vesicles promotes selective regeneration in ischemic hearts. Exp Mol Med. 2021;53:695-708.

14. Luo H, Zhu G, Xu J, Lai $Q$, Yan B, Guo Y, et al. HOTTIP IncRNA promotes hematopoietic stem cell self-renewal leading to aml-like disease in mice. Cancer Cell. 2019;36:645-59. e648

15. Zhang $Y$, Li Y, Hu Q, Xi Y, Xing Z, Zhang Z, et al. The IncRNA H19 alleviates muscular dystrophy by stabilizing dystrophin. Nat Cell Biol. 2020;22:1332-45.

16. Li G, Kryczek I, Nam J, Li X, Li S, Li J, et al. LIMIT is an immunogenic IncRNA in cancer immunity and immunotherapy. Nat Cell Biol. 2021;23:526-37.

17. Daneshvar K, Ardehali MB, Klein IA, Hsieh FK, Kratkiewicz AJ, Mahpour A, et al. InCRNA DIGIT and BRD3 protein form phase-separated condensates to regulate endoderm differentiation. Nat Cell Biol. 2020;22:1211-22.

18. Mo S, Zhang L, Dai W, Han L, Wang R, Xiang W, et al. Antisense IncRNA LDLRAD4AS1 promotes metastasis by decreasing the expression of LDLRAD4 and predicts a poor prognosis in colorectal cancer. Cell Death Dis. 2020;11:155.

19. Li Y, Yan G, Zhang J, Chen W, Ding T, Yin Y, et al. LncRNA HOXA11-AS regulates calcium oxalate crystal-induced renal inflammation via miR-124-3p/MCP-1. J Cell Mol Med. 2020;24:238-49.

20. Huang Z, Zhou JK, Peng Y, He W, Huang C. The role of long noncoding RNAs in hepatocellular carcinoma. Mol Cancer. 2020;19:77.

21. Zhang $Q$, Cheng $Q$, Xia $M$, Huang $X$, He X, Liao J. Hypoxia-induced IncRNANEAT1 sustains the growth of hepatocellular carcinoma via regulation of miR199a-3p/UCK2. Front Oncol. 2020;10:998.

22. Zhu XT, Yuan JH, Zhu TT, Li YY, Cheng XY. Long noncoding RNA glypican 3 (GPC3) antisense transcript 1 promotes hepatocellular carcinoma progression via epigenetically activating GPC3. FEBS J. 2016;283:3739-54.

23. Pu J, Wang J, Wei H, Lu T, Wu X, Wu Y, et al. IncRNA MAGI2-AS3 prevents the development of HCC via recruiting KDM1A and promoting H3K4me2 demethylation of the RACGAP1 promoter. Mol Ther Nucleic Acids. 2019;18:351-62. 
24. Klingenberg M, Gross $M$, Goyal A, Polycarpou-Schwarz M, Miersch T, Ernst AS, et al. The long noncoding RNA cancer susceptibility 9 and RNA binding protein heterogeneous nuclear ribonucleoprotein I form a complex and coregulate genes linked to Akt signaling. Hepatology. 2018;68:1817-32.

25. Yuan JH, Yang F, Wang F, Ma JZ, Guo YJ, Tao QF, et al. A long noncoding RNA activated by TGF-beta promotes the invasion-metastasis cascade in hepatocellular carcinoma. Cancer Cell. 2014;25:666-81.

26. Wang G, Wang Q, Liang N, Xue H, Yang T, Chen X, et al. Oncogenic driver genes and tumor microenvironment determine the type of liver cancer. Cell Death Dis. 2020;11:313.

27. Sharma A, Seow JJW, Dutertre CA, Pai R, Bleriot C, Mishra A, et al. Onco-fetal reprogramming of endothelial cells drives immunosuppressive macrophages in hepatocellular carcinoma. Cell. 2020;183:377-94. e321

28. Meng G, Li B, Chen A, Zheng M, Xu T, Zhang H, et al. Targeting aerobic glycolysis by dichloroacetate improves Newcastle disease virus-mediated viro-immunotherapy in hepatocellular carcinoma. Br J Cancer. 2020;122:111-20.

29. Calderaro J, Rousseau B, Amaddeo G, Mercey M, Charpy C, Costentin C, et al. Programmed death ligand 1 expression in hepatocellular carcinoma: Relationship With clinical and pathological features. Hepatology. 2016;64:2038-46.

30. Cassetta L, Fragkogianni S, Sims AH, Swierczak A, Forrester LM, Zhang $H$, et al. Human tumor-associated macrophage and monocyte transcriptional landscapes reveal cancer-specific reprogramming, biomarkers, and therapeutic targets. Cancer Cell. 2019;35:588-602. e510

31. Xing X, Wang Y, Zhang X, Gao X, Li M, Wu S, et al. Matrix stiffness-mediated effects on macrophages polarization and their LOXL2 expression. FEBS J. 2021;288:3465-77.

32. Ding $T$, Xu J, Wang $F$, Shi M, Zhang $Y$, Li SP, et al. High tumor-infiltrating macrophage density predicts poor prognosis in patients with primary hepatocellular carcinoma after resection. Hum Pathol. 2009;40:381-9.

33. Natoli M, Herzig P, Pishali Bejestani E, Buchi M, Ritschard R, Lloyd GK, et al. Plinabulin, a distinct microtubule-targeting chemotherapy, promotes $\mathrm{m} 1$-like macrophage polarization and anti-tumor immunity. Front Oncol. 2021;11:644608.

34. Shigeta K, Datta M, Hato T, Kitahara S, Chen IX, Matsui A, et al. Dual programmed death receptor-1 and vascular endothelial growth factor receptor-2 blockade promotes vascular normalization and enhances antitumor immune responses in hepatocellular carcinoma. Hepatology. 2020;71:1247-61.

35. Zhu Y, Yang J, Xu D, Gao XM, Zhang Z, Hsu JL, et al. Disruption of tumourassociated macrophage trafficking by the osteopontin-induced colony-stimulating factor-1 signalling sensitises hepatocellular carcinoma to anti-PD-L1 blockade. Gut. 2019;68:1653-66.

36. Hu ZQ, Zhou SL, Li J, Zhou ZJ, Wang PC, Xin HY, et al. Circular RNA Sequencing Identifies CircASAP1 as a Key Regulator in Hepatocellular Carcinoma Metastasis. Hepatology. 2020;72:906-22.

37. Yuan JH, Liu XN, Wang TT, Pan W, Tao QF, Zhou WP, et al. The MBNL3 splicing factor promotes hepatocellular carcinoma by increasing PXN expression through the alternative splicing of IncRNA-PXN-AS1. Nat Cell Biol. 2017;19:820-32.

38. Tang Z, Li C, Kang B, Gao G, Li C, Zhang Z. GEPIA: a web server for cancer and normal gene expression profiling and interactive analyses. Nucleic Acids Res. 2017;45:W98-W102.

39. Liu Q, He L, Li S, Li F, Deng G, Huang X, et al. HOMER3 facilitates growth factormediated beta-Catenin tyrosine phosphorylation and activation to promote metastasis in triple negative breast cancer. J Hematol Oncol. 2021;14:6.

40. Gyori DS, Mocsai A. Osteoclast signal transduction during bone metastasis formation. Front Cell Dev Biol. 2020;8:507.

41. Srivastava S, Furlan SN, Jaeger-Ruckstuhl CA, Sarvothama M, Berger C, Smythe KS et al. Immunogenic chemotherapy enhances recruitment of CAR-T cells to lung tumors and improves antitumor efficacy when combined with checkpoint blockade. Cancer Cell. 2021:39:193-208. e110

42. Kaymak I, Williams KS, Cantor JR, Jones RG. Immunometabolic Interplay in the Tumor Microenvironment. Cancer Cell. 2021;39:28-37.

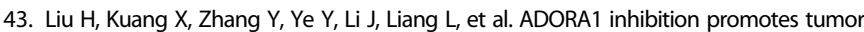
immune evasion by regulating the ATF3-PD-L1 Axis. Cancer Cell. 2020;37:324-39. e328

44. Montagner M, Bhome R, Hooper S, Chakravarty P, Qin X, Sufi J, et al. Crosstalk with lung epithelial cells regulates Sfrp2-mediated latency in breast cancer dissemination. Nat Cell Biol. 2020;22:289-96.

45. Zhu AX, Finn RS, Edeline J, Cattan S, Ogasawara S, Palmer D, et al. Pembrolizumab in patients with advanced hepatocellular carcinoma previously treated with sorafenib (KEYNOTE-224): a non-randomised, open-label phase 2 trial. Lancet Oncol. 2018;19:940-52.

46. El-Khoueiry AB, Sangro B, Yau T, Crocenzi TS, Kudo M, Hsu C, et al. Nivolumab in patients with advanced hepatocellular carcinoma (CheckMate 040): an openlabel, non-comparative, phase 1/2 dose escalation and expansion trial. Lancet. 2017;389:2492-502.

47. Yau T, Kang YK, Kim TY, El-Khoueiry AB, Santoro A, Sangro B, et al. Efficacy and Safety of Nivolumab Plus Ipilimumab in Patients With Advanced Hepatocellular
Carcinoma Previously Treated With Sorafenib: The CheckMate 040 Randomized Clinical Trial. JAMA Oncol. 2020;6:e204564.

48. Yu, AT, Berasain, C, Bhatia, S, Rivera, K, Liu, B, Rigo, F et al. PHAROH IncRNA regulates Myc translation in hepatocellular carcinoma via sequestering TIAR. Elife (2021);10.

49. Li JK, Chen C, Liu JY, Shi JZ, Liu SP, Liu B, et al. Long noncoding RNA MRCCAT1 promotes metastasis of clear cell renal cell carcinoma via inhibiting NPR3 and activating p38-MAPK signaling. Mol Cancer. 2017;16:111.

50. Zeng T, Tang Z, Liang L, Suo D, Li L, Li J, et al. PDSS2-Del2, a new variant of PDSS2, promotes tumor cell metastasis and angiogenesis in hepatocellular carcinoma via activating NF-kappaB. Mol Oncol. 2020;14:3184-97.

51. Tian X, Wu Y, Yang Y, Wang J, Niu M, Gao S, et al. Long noncoding RNA LINC00662 promotes M2 macrophage polarization and hepatocellular carcinoma progression via activating Wnt/beta-catenin signaling. Mol Oncol. 2020;14:462-83.

52. Garcia-Lezana T, Lopez-Canovas JL, Villanueva A. Signaling pathways in hepatocellular carcinoma. Adv Cancer Res. 2021;149:63-101.

53. He Y, Huang H, Jin L, Zhang F, Zeng M, Wei L, et al. CircZNF609 enhances hepatocellular carcinoma cell proliferation, metastasis, and stemness by activating the Hedgehog pathway through the regulation of miR-15a-5p/15b-5p and GLI2 expressions. Cell Death Dis. 2020;11:358.

54. Song M, Pan Q, Yang J, He J, Zeng J, Cheng S, et al. Galectin-3 favours tumour metastasis via the activation of beta-catenin signalling in hepatocellular carcinoma. Br J Cancer. 2020;123:1521-34

55. Petty AJ, Li A, Wang X, Dai R, Heyman B, Hsu D, et al. Hedgehog signaling promotes tumor-associated macrophage polarization to suppress intratumoral CD8+ T cell recruitment. J Clin Invest. 2019;129:5151-62.

\section{ACKNOWLEDGEMENTS}

This work was supported by Special Funding for Guangxi Special Experts, Guangxi Medical High-level Leading Talents Training "139" Project, and Baise Scientific Research and Technology Planning Bureau (BK20204723).

\section{AUTHOR CONTRIBUTIONS}

$J P, H W, Q T$, and $W L$ designed the idea and experiments. JP, YZ, AW, ZQ, ZX, WL, and YL performed the experiments. JP, HW, QT, and JW analyzed the data. JP and HW are the major contributors in writing the manuscript. All authors read and approved the final paper.

\section{COMPETING INTERESTS}

The authors declare no competing interests.

\section{ADDITIONAL INFORMATION}

Supplementary information The online version contains supplementary material available at https://doi.org/10.1038/s41419-021-04309-z.

Correspondence and requests for materials should be addressed to Qianli Tang or Huamei Wei.

Reprints and permission information is available at http://www.nature.com/reprints

Publisher's note Springer Nature remains neutral with regard to jurisdictional claims in published maps and institutional affiliations.

\begin{abstract}
Open Access This article is licensed under a Creative Commons Attribution 4.0 International License, which permits use, sharing, adaptation, distribution and reproduction in any medium or format, as long as you give appropriate credit to the original author(s) and the source, provide a link to the Creative Commons license, and indicate if changes were made. The images or other third party material in this article are included in the article's Creative Commons license, unless indicated otherwise in a credit line to the material. If material is not included in the article's Creative Commons license and your intended use is not permitted by statutory regulation or exceeds the permitted use, you will need to obtain permission directly from the copyright holder. To view a copy of this license, visit http://creativecommons.org/licenses/by/4.0/.
\end{abstract}

(c) The Author(s) 2021 